\title{
Compact, Slotted, Printed Antennas for Dual-Band Communication in Future Wireless Sensor Networks
}

\author{
Constantine G. Kakoyiannis and Philip Constantinou \\ Mobile Radio Communications Laboratory, National Technical University of Athens, Zographos Polytechnic Campus, \\ 9 Iroon Polytechniou Street, 15773 Athens, Greece
}

Correspondence should be addressed to Constantine G. Kakoyiannis; kkak@mobile.ntua.gr

Received 13 August 2012; Revised 25 October 2012; Accepted 20 December 2012

Academic Editor: Fan Yang

Copyright (C) 2013 C. G. Kakoyiannis and P. Constantinou. This is an open access article distributed under the Creative Commons Attribution License, which permits unrestricted use, distribution, and reproduction in any medium, provided the original work is properly cited.

\begin{abstract}
Inverted-F antennas (IFAs) are a primary choice to implement the radiating system of portable devices. A tried and tested idea can remain topical if proven useful in modern applications. This paper shows that printed IFAs (PIFAs) are capable of forming robust, compact, dual-band radiating systems for wireless microsensors with an adjustable spacing between bands. Reactive tuning was applied by inductively loading the structures with prefractal slots; inductive slot loading degenerates higher-order resonances and increases the fractional bandwidth (FBW). The current distributions revealed that most of the element area is used for radiation at both resonances. In radiation terms, the antennas provide satisfactory gains and high efficiencies $(\geq 82 \%)$. A simple figure of merit is used to compare the performance of the three PIFAs head to head. Operation at $2.5 \mathrm{GHz}$ and $5.5 \mathrm{GHz}$ indicated that changes in slot geometry almost double the FBW. The proposed antennas serve both the $5.15-5.35 \mathrm{GHz}$ U-NII and the $5.8 \mathrm{GHz}$ ISM bands; at the lower band, their size is less or equal to the half-wavelength dipole. This study of dual-band antennas also showed that the aggregate FBW of a PIFA is bounded; by degenerating higher-order modes, the designer redistributes whatever bandwidth is available by the antenna itself to the desired bands.
\end{abstract}

\section{Introduction}

1.1. Background and Motivation. For the past fifteen years inverted-F antennas have been a major design vehicle for the implementation of radiating systems for portable devices [1-8]. IFAs use image theory as their basic miniaturization technique through a shorting wall or pin that ties them to the ground plane (GNDP). The planar version of IFAs inherits the cavity features of microstrip patch antennas, thus its bandwidth is inherently limited. Extensive antenna and/or GNDP alteration has been applied through slots and slits to excite more radiating modes. The printed version of IFAs is not shaped like a cavity, thus it is significantly more broadband.

Dual-band printed and planar IFAs have drawn significant attention in the existing literature [9-21]. Starting from legacy applications like PCMCIA cards [9], the use of dualband PIFAs was extended to (i) wireless LAN communication for laptop computers in the $2.4-$ and $5.2 / 5.8-\mathrm{GHz}$ bands
[11-13, 15, 16, 20], (ii) mobile phone antennas operating at $900 / 1900 \mathrm{MHz}$ [14], (iii) dual-band communication at 2.6 and $5.5 \mathrm{GHz}$ [17], (iv) mobile phones equipped with WLAN capability operating at DCS/ISM frequencies [18], and (v) dualband antennas printed on USB dongles serving as multiband antennas [19]. In the case of portable computers (laptops), printed antennas are usually installed under the lid on the back side of the screen [20]. Furthermore, printed dual-band antennas can be readily applied to WLAN routers. Finally, in a recent study, Li et al. [21] proposed a reconfigurable structure that can be transformed from a double planar IFA into a meandered loop antenna by means of a PIN diode switch. The two modes of operation enable the antenna to achieve complementary operating bands in the $0.79-2.56 \mathrm{GHz}$ range.

This paper addresses the design of dual-band PIFAs for wireless sensor nodes. It aims to show that by properly applying inductive loading in the form of prefractal slots, printed IFAs are capable of producing robust, compact, dualband radiating systems for wireless microsensors with an 
adjustable spacing between bands. One could argue that dual-band antennas find no use in wireless sensor networks (WSNs), because dual-band WSNs do not exist: the second integrated transceiver that is required along with its peripheral passive components bring extra complexity, cost, and energy consumption for a wireless microsensor. So, it is best to wait until very large scale integration (VLSI) technology matures to the point of single-chip, dual-band, integrated transceivers. However, the above arguments do not hold for two reasons. On one hand, dual-band communication is inherent in real-world WSN deployments: the microsensors implement various algorithms, for example, routing, localization, virtual array formation, and improved connectivity and coverage. These algorithms depend on GPS signal reception; hence, a second communication band comes into play. On the other hand, dual-band, single-chip, integrated transceivers are already here: Cho et al. [22] designed an energy-efficient, dual-band, fully integrated transceiver for body sensor network applications in the $30-70 \mathrm{MHz}$ and MICS (402-405 MHz) bands. Thus, it is anticipated that dualband communication will become vital in future wireless sensor networks.

To the best of the authors' knowledge, the only existing study on dual-band antennas that target wireless sensors is the work of Mendes et al. [10]. We comment on this work in Section 4 , along with the rest of the relevant literature.

1.2. Theoretical Considerations. Boyle $[5,23]$ and Boyle and Ligthart [24] used superposition and divided the operation of the antenna into the balanced (nonradiating) and the radiating mode. One of their key findings was that the PCB length has a strong effect on antenna quality factor, $Q$ : the $Q$ is minimized when the length is close to the $\lambda / 2$ - or $\lambda$ antiresonance. Hence, the bandwidth (BW), which is related to the quality factor $Q$, is strongly affected by the length of the GNDP. Furthermore, in the case of dual-band PIFAs, the width of the two bands is not independent; to improve BW at the lower resonance, the higher resonance $\mathrm{BW}$ must be degraded.

Referring to the internal workings of dual-band slotted planar IFAs $[1,25]$, Boyle suggested that slots in the antenna top plate between the feed and short pins allow independent variation of the impedance transformation and shunt reactance $[5,23,24]$. Furthermore, slots in the top plate can tune a planar inverted-L antenna (ILA) that is naturally resonant between the lower and upper resonance. If the slot is too long, the lower BW is degraded; if the slot is too short, the upper BW is degraded.

The technique of degenerating higher-order radiating modes by inductive loading of the antenna with slots applies equally well in the case of nonadjacent frequency bands. This has been shown for planar IFAs by Liu et al. [25] and Boyle and Ligthart [23, 24]. Angelopoulos et al. [9] showed that this response is also feasible with printed IFAs: the authors of [9] presented an application of the slot-loading concept, which involved a dual-band PIFA comprised of two $\lambda / 4$ monopoles coupled and separated by an L-slot. It was suggested that each monopole radiates in its own resonance frequency $(2.45 / 5.25 \mathrm{GHz})$ when fed by a single feeding port connected to the smaller monopole. It was also theorized that the function of the L-slot was to form a gap for higher frequency currents, which at the same time is not wide enough to disturb the lower resonance. The work described herein will demonstrate that, in general, this is not the case.

1.3. Scope and Structure of the Paper. Based on our experience with multimode, single-band printed IFAs [26], we have attempted to design microsensor-oriented PIFAs which should satisfy the following specifications.

(1) Display a bandwidth at least equal to $100 \mathrm{MHz}$ in the vicinity of $2.5 \mathrm{GHz}$, thus a fractional bandwidth $\mathrm{FBW}_{V \ell} \geq 4 \%$.

(2) Display a bandwidth at least equal to $200 \mathrm{MHz}$ at the lower end of the 5-6 GHz range. A good application example is the U-NII $5.15-5.35 \mathrm{GHz}$ band, which features a fractional bandwidth $\mathrm{FBW}_{V u}=3.8 \%$.

(3) Achieve the previous two specifications without exceeding the electrical size, $k a$, of the half-wavelength dipole, $(k a)_{\lambda / 2}=\pi / 2 \mathrm{rad}$, at the lower band.

(4) Achieve a mean total radiation efficiency over both bands at least equal to $80 \%$, that is, $\overline{n_{\text {total }}} \geq-1 \mathrm{~dB}$.

The paper describes the design of printed inverted-F antennas that have been inductively loaded by four different pre-fractal slots. The first three slot topologies were discussed in detail in [26]: they are the simple, generalized, and dual generalized, Koch curves of the second iteration. The fourth slot resembles the quadratic type 2 Koch curve; its properties are described in Section 3.4. The effect of the size of the ground plane on both frequency bands was also investigated. The reactive loading imposed by the pre-fractal slots not only surpassed the specifications, but also outperformed the results of several prior studies. Section 2 discusses design and modelling issues. Section 3 displays an array of numerical results produced by accurate computational models of the four slotted antenna configurations. Section 4 discusses the results. Section 5 presents measurement results harvested from a prototype antenna. Finally, Section 6 concludes the paper.

\section{Antenna Design and Electromagnetic Modelling}

Out of the four antennas that were designed, the first three were inductively loaded by slots shaped after the following pre-fractal curves:

(1) the Koch curve of the second iteration (abbreviated herein as "K2") [26],

(2) the generalized Koch curve of the second iteration (abbreviated herein as "GK2") [26], and

(3) the dual generalized Koch curve of the second iteration (abbreviated herein as "D-GK2") [26].

Apart from [26], the properties of Koch curve variants are also described in [27-30], thus the details are omitted here for 


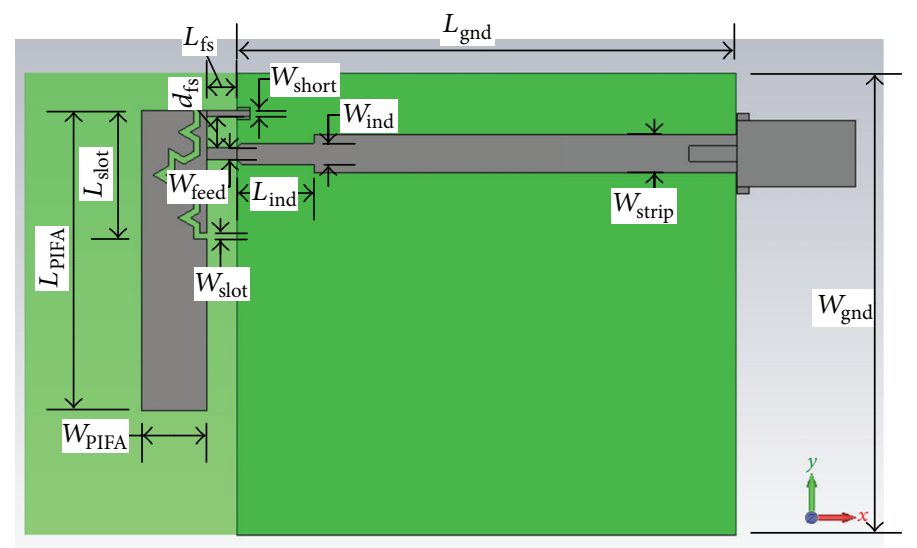

FIGURE 1: Parametric breakdown of the printed IFA loaded with an embedded Koch-shaped slot. The substrate is transparent, showing the ground plane on the bottom layer. The nomenclature of parameters is a simplified version of the one used in [26].

brevity. The fourth antenna was loaded by a quadratic type 2 Koch curve of the second iteration (abbreviated herein as "MK2"), which is described in Section 3.4.

The antennas were designed to operate about the centre frequencies $f_{0 \ell}=2.5 \mathrm{GHz}$ and $f_{0 u}=5.25 \mathrm{GHz}$, where the corresponding wavelengths are $\lambda_{0 \ell}=120 \mathrm{~mm}$ and $\lambda_{0 u} \simeq 57.1$ $\mathrm{mm}$. The 2-layer PCBs are made of Taconic TRF- 45 material $\left(\varepsilon_{r}=4.38\right.$ and $\tan \delta_{e}=0.0028$ at $\left.2.5 \mathrm{GHz}\right)$. The substrate is $H_{\text {sub }}=1.63 \mathrm{~mm}$ thick, and it is loaded with $1 \mathrm{oz}$ copper on each side [31]. Higher frequencies at the upper band demand the use of a good quality substrate that features low-loss and well-controlled relative permittivity. TRF- 45 combines low-loss with an FR4-compatible printing process. In this system model, the size of the PCB represents the whole size of the sensor node. On the top layer, we etched the printed antenna and the $3.1 \mathrm{~mm}$-wide microstrip line that excites it. On the bottom layer, there is a continuous copper cladding that serves as the ground plane (GNDP) of both antenna and microstrip line. The GNDP was removed below the antenna. Assuming that the PCB in Figure 1 is an autonomous node, then all radiofrequency $(\mathrm{RF})$ and baseband integrated circuits and discrete components would be soldered on the bottom layer in an actual implementation. This structure can be easily transformed to uniplanar (single-sided $\mathrm{PCB}$ ) by changing the feed to coplanar waveguide (CPW). The use of groundbacked CPW would double the available space for RF and baseband electronics.

The antennas were designed and simulated by means of a transient solver, which is part of a full-wave E/M simulator that uses the finite integration technique (FIT) to reformulate Maxwell's integral equations into the so-called "Maxwell Grid Equations." In the time domain, by applying Yee's spatial discretization and time-stepping scheme, FIT results in the same set of equations as FDTD $[32,33]$. The structures were excited by a wideband Gaussian pulse (DC$7 \mathrm{GHz}$ ). A spatially adaptive hexahedral mesh discretized the objects. Finer meshing was applied inside the substrate to capture the large gradients of the E-field; the same applies across the microstrip feed, on the main arm, and inside the dielectric of the SMA connector. The simulator stopped when the initial system energy decayed by $40 \mathrm{~dB}$. This tradeoff between simulation speed and FFT truncation error was slightly in favour of speed. The maximum cell size at $7 \mathrm{GHz}$ (smallest wavelength) was set at $\lambda_{7 \mathrm{GHz}} / 20$. The solvable space was terminated at four Berenger PML layers, with a distance-to-boundary equal to $\lambda_{3 \mathrm{GHz}} / 8$. The antennas did not exhibit topological symmetry, thus did not satisfy the required boundary conditions of electric and magnetic flux for the placement of "magnetic walls", which would lower the computational burden. The complexity of the models varied in the range $370-495 \times 10^{3}$ Yee cells. Complexity depends on the size of the PCB and the level of detail exhibited by the antenna element. Narrow copper traces and narrow object spacing contribute greatly to complexity. The MK2 model exhibited $28-33 \%$ greater complexity compared to the other three model due to its inherently finer structure. Simulation setup settings are summarized in Table 1 .

\section{Proposed Radiating Structures and Numerical Results}

The design of the four slotted antennas is based on the manual optimization procedure and design guidelines that were reported in [26]. The controlling parameters of the antennas converged to the values listed in Table 2. The nomenclature of parameters shown in Figure 1 is a representative of all four structures.

Table 2 lists at the bottom parameters $L_{\text {ind }}$ and $W_{\text {ind }}$. In order to achieve a well-balanced input impedance in both operating bands, there is a need to compensate the capacitive part of the impedance. The imaginary part, $X_{\text {in }}(j \omega)$, assumed large negative values, because the common length of the feeding and shorting stubs, $L_{\mathrm{fs}}$, is short due to high-frequency upper band, thus the main arm of the PIFA approaches the ground plane far more than it did in the case of the multimode, single-band counterparts [26]. To mend this problem, the last segment of the microstrip feed line, whose length equals $L_{\text {ind }}$, was narrowed from $W_{\text {strip }}$ down to $W_{\text {ind }}$. The 

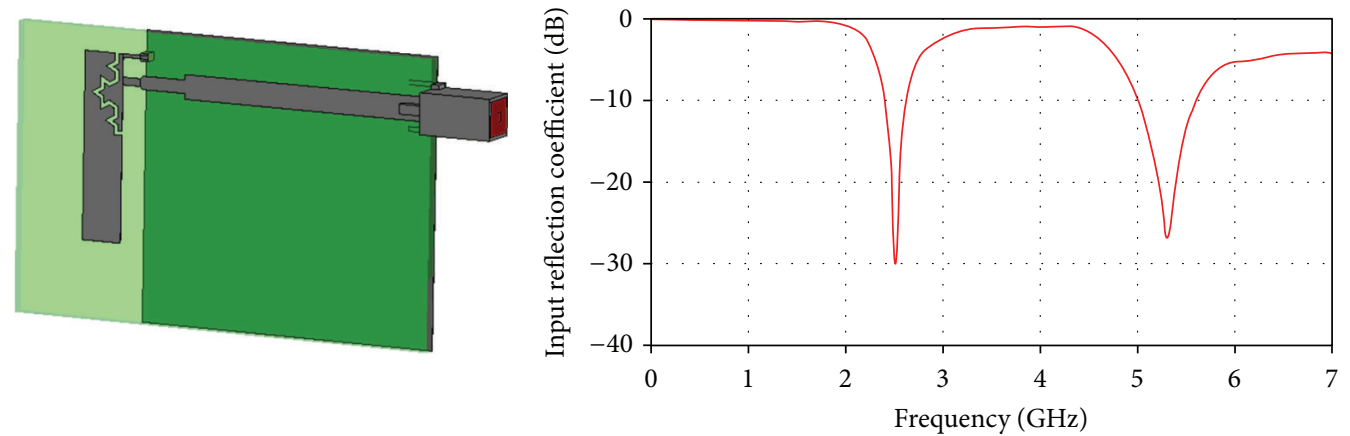

FIgURE 2: The computational model of the dual-band K2 PIFA in perspective and the magnitude of its input reflection coefficient in the range DC-7 GHz.

TABLE 1: Settings applied to the FIT-TS solver $[32,33]$ during the simulations of the slotted PIFAs.

\begin{tabular}{lc}
\hline \multicolumn{2}{c}{ Slotted PIFA simulation setup } \\
\hline Excitation signal & Gaussian pulse, DC-7 GHz \\
Spatial discretization & Hexahedral, nonuniform \\
Convergence condition & $40 \mathrm{~dB}$ energy decay \\
Maximum cell size at $f_{\max }$ & $\lambda_{\min } / 20$ \\
Absorbing boundary conditions & 4 -layer PML \\
Minium distance to PML & $\lambda_{3 \mathrm{GHz}} / 8$ \\
boundary & $(370-385) \times 10^{3}$ Yee cells \\
Computational complexity, & \\
(D-) $(\mathrm{G})$ K2 & $495 \times 10^{3}$ Yee cells \\
Computational complexity, MK2
\end{tabular}

narrowed segment inserts extra inductance in series with $Z_{\text {in }}(j \omega)$ and makes up the simplest matching circuit.

It is also noted that the design procedure verified the manual optimization algorithm given in [26], but with one important difference: excluding parameters $L_{\text {PIFA }}$ and $L_{\text {slot }}$, the other six parameters affect the response of the antenna in the two frequency bands in opposite ways. For example, a change in $d_{\mathrm{fs}}$ that improves matching and resonance in the lower band, worsens the performance in the upper band, and vice versa. Inevitably, parameter values were chosen in a compensatory manner.

During simulations for the virtual prototyping, an automated calculation of the electrical size of antennas was required. Thus, the automated estimation of the radius of the circumscribing sphere was based on the simplified expression

$$
a=\frac{1}{2} \sqrt{W_{\text {strip }}^{2}+\left(W_{\mathrm{PIFA}}+L_{\mathrm{fs}}+L_{\mathrm{gnd}}\right)^{2}}
$$

which produces slightly larger electrical sizes than the actual ones.

This section demonstrates through accurate computational models (virtual prototypes) the electrical performance of the four slotted PIFAs.
3.1. Dual-Band PIFA Loaded by the Koch Slot. The first antenna that was designed is inductively loaded by a Kochshaped slot of the second iteration, which has been described in [26] and also in [27-30]. The computational model and the broadband magnitude of the input reflection coefficient are depicted in Figure 2. Antenna dimensions, starting with $L_{\text {slot }}$, were adjusted so as to obtain resonances at the desired bands. The high-frequency upper band leads to a significantly smaller slot compared to the one in [26]: the side length ratio of the outline of the $\mathrm{K} 2$ slot remains constant as dimensions vary, thus the slot is much shorter, allowing for the design of a PIFA with a significantly narrower main arm. The feeding line is offset with respect to the longitudinal symmetry axis of the PCB. Application of the offset feed further facilitates matching and resonance in both bands. The key numerical results of the K2 PIFA are listed in Table 4. The antenna meets all four specifications, but the figure of merit assumes negative values in decibels. In the lower band the low FoM values are due to the reduced bandwidth, whereas in the upper band they result from the increased electrical size: in the $5-6 \mathrm{GHz}$ range the K2 PIFA is electrically large ( $k a>2 \mathrm{rad}$ ).

The Smith charts in Figure 3 were derived after placing the reference plane of the scattering parameters at the beginning of the SMA end launch connector. The charts indicate that the compensatory dimensions that were chosen for the $\mathrm{K} 2$ antenna produce a frequency response that is well-balanced between inductive and capacitive reactance. Furthermore, in the lower band the input resistance is equally distributed below and above $50 \Omega$, whereas in the upper band we mostly get $R_{\text {in }}<50 \Omega$.

The surface current distributions (SCDs) in Figure 4 show concurrent maxima of current density at every point on the PCB. Concurrent maxima are physically unrealisable due to the phase shift along the structure, but they are useful for identifying "hot" and "cold" regions. The SCDs indicate that (i) in the lower frequency band practically the whole extent of the main arm radiates and (ii) in the upper band the main arm radiates mainly along the $\mathrm{K} 2$ slot, whereas a discontinuous image forms on the ground plane with a greater overall length. In both bands, return currents are strong along all four sides of the GNDP. 


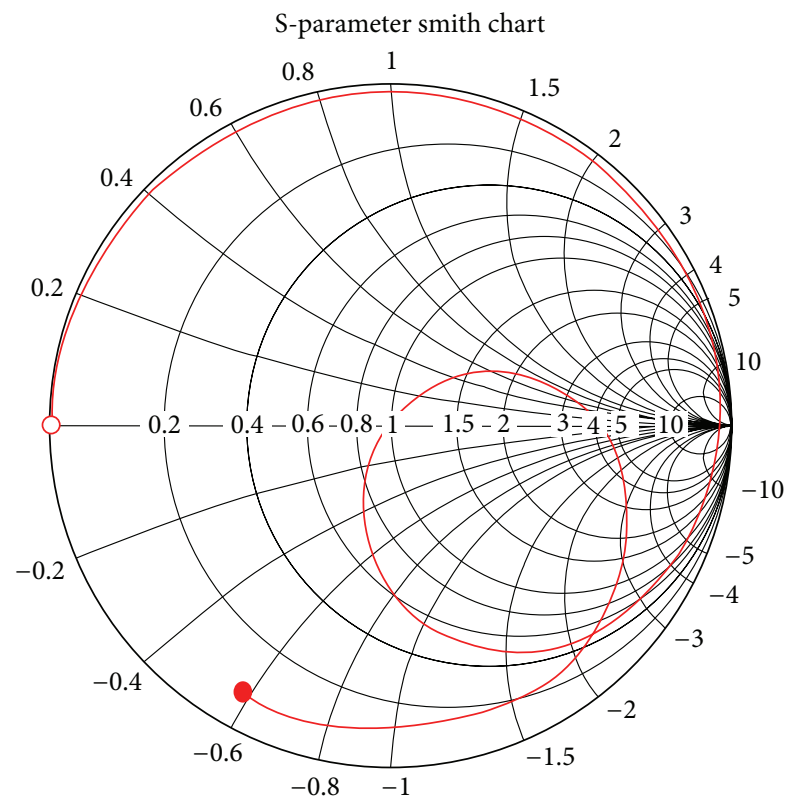

○ $0(-1.876 e-031,-3.063 e-015) \mathrm{ohm}$

- $4(3.627,-29.28) \mathrm{ohm}$

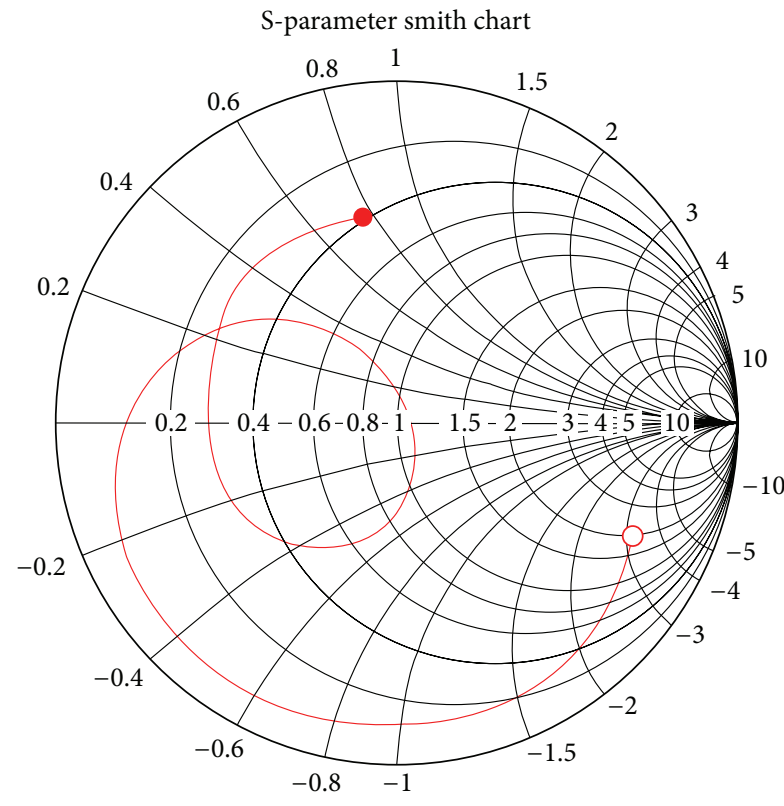

○ $3(96.33,-162.1) \mathrm{ohm}$

- $7(19.94,38.3) \mathrm{ohm}$

(a)

(b)

FIgURE 3: Smith chart of the complex reflection coefficient of the dual-band K2 PIFA in the overlapping frequency ranges DC-4 GHz and 3-7 GHz.

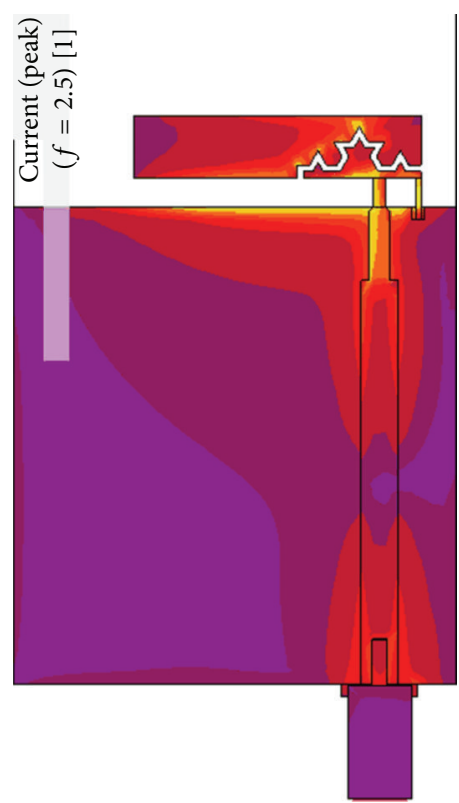

$(\mathrm{A} / \mathrm{m})$

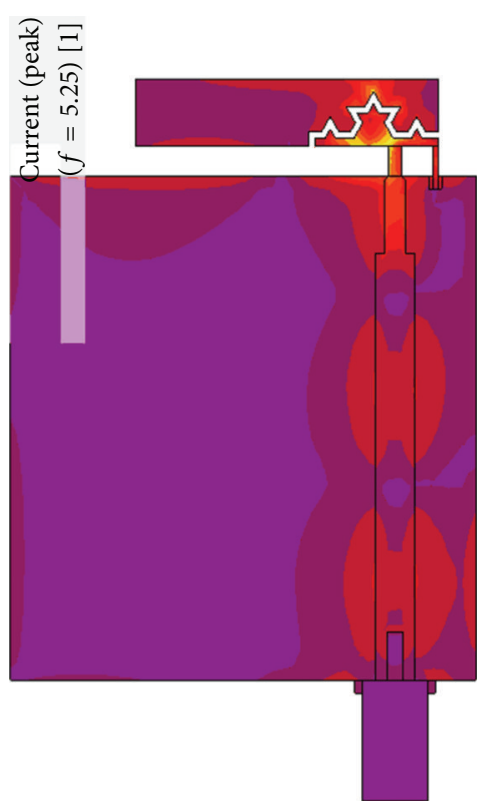

$(\mathrm{A} / \mathrm{m})$

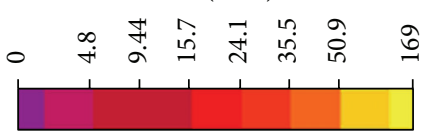

(a)

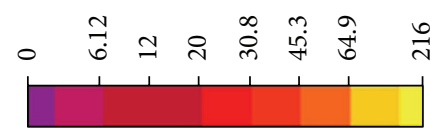

(b)

FIGURE 4: Surface current distributions of the dual-band K2 PIFA at $2.5 \mathrm{GHz}$ and $5.25 \mathrm{GHz}$. 
TABLE 2: Values of parameters that determine the design of the four proposed dual-band PIFAs. All dimensions are in millimetres.

\begin{tabular}{lcccc}
\hline Dimension & K2 & GK2 & D-GK2 & MK2 \\
\hline$L_{\text {sub }} \times W_{\text {gnd }}$ & $57 \times 37$ & $54 \times 35.8$ & $54 \times 36.2$ & $60 \times 33.6$ \\
$L_{\text {gnd }} \times W_{\text {gnd }}$ & $40 \times 37$ & $40 \times 35.8$ & $40 \times 36.2$ & $40 \times 33.6$ \\
$L_{\text {PIFA }} \times\left(W_{\text {PIFA }}+L_{\text {fs }}\right)$ & $24.0 \times 7.7$ & $23.3 \times 7.8$ & $23.2 \times 7.6$ & $21.2 \times 8.1$ \\
$W_{\text {strip }}$ & 3.1 & 3.1 & 3.1 & 3.1 \\
$d_{\text {fs }}$ & 2.5 & 2.1 & 2.2 & 1.5 \\
$L_{\text {slot }}$ & 10.3 & 8.9 & 8.9 & 6.7 \\
$L_{\text {PIFA }}$ & 24.0 & 23.3 & 23.2 & 21.6 \\
$L_{\text {fs }}$ & 2.4 & 1.4 & 1.5 & 1.5 \\
$W_{\text {slot }}$ & 0.5 & 0.5 & 0.6 & 0.3 \\
$W_{\text {PIFA }}$ & 5.3 & 6.4 & 6.1 & 6.6 \\
$W_{\text {short }}$ & 0.5 & 0.4 & 0.4 & 0.4 \\
$W_{\text {feed }}$ & 1.0 & 1.1 & 0.6 & 0.9 \\
$L_{\text {ind }}$ & 6.2 & 6.1 & 6.4 & 6.2 \\
$W_{\text {ind }}$ & 1.7 & 1.9 & 2.3 & 1.9 \\
\hline
\end{tabular}

The first row denotes the extent of the PCB, the second row denotes the size of the ground plane, and the third one denotes the extent of the antenna element.

3.2. Dual-Band PIFA Loaded by the Generalized Koch Slot. The second antenna that was designed is inductively loaded by a generalized Koch-shaped slot of the second iteration, which has been described in [26]. The computational model and the broadband magnitude of the input reflection coefficient are depicted in Figure 5. The antenna resonates at 2.5 GHz, $5.14 \mathrm{GHz}$, and $5.71 \mathrm{GHz}$. This means that by a simple change in slot geometry the reactive loading produces a second $S_{11}$ minimum in the 5-6 GHz range. As a result, the 5$6 \mathrm{GHz}$ range meets the VSWR criterion practically in its full extent. The key numerical results of the K2 PIFA are listed in Table 4.

The Smith charts in Figure 6 indicate that the compensatory dimensions that were chosen for the GK2 antenna produce a frequency response that is well-balanced between inductive and capacitive reactance. Furthermore, in the lower band the input resistance is equally distributed below and above $50 \Omega$, whereas in the upper band we mostly get $R_{\text {in }}<$ $50 \Omega$. Finally, the $S_{11}$ minimum at $5.71 \mathrm{GHz}$ is not a resonance in the strict sense, but appears as a result of the small loop that the curve traces.

The surface current distributions in Figure 7 show concurrent maxima of current density at every point on the PCB. In the lower frequency band, the same comments as in the K2 case apply. At $5.14 \mathrm{GHz}$, the main arm radiates mostly along the GK2 slot, whereas a discontinuous image forms on the ground plane with greater overall length. At $5.71 \mathrm{GHz}$, the main arm radiates uniformly (not focused around the slot), whereas a strong image forms on the ground plane away from the slot.

3.3. Dual-Band PIFA Loaded by the Dual Generalized Koch Slot. The third antenna was inductively loaded by the dual generalized Koch-shaped slot of the second iteration, which has been described in [26]. This particular version of the
Koch pre-fractal shape exhibits lower variations in height and thus produced an antenna with slightly narrower main arm. The computational model and the broadband magnitude of the input reflection coefficient are depicted in Figure 8. The antenna resonates at $2.5 \mathrm{GHz}, 5.11 \mathrm{GHz}$, and $5.77 \mathrm{GHz}$, that is, the dual of the generalized Koch slot preserves the second $S_{11}$ minimum in the 5-6 GHz range. The key numerical results of the K2 PIFA are listed in Table 4.

The Smith charts in Figure 9 indicate that the compensatory dimensions that were chosen for the D-GK2 antenna produce a frequency response that is well-balanced between inductive and capacitive reactance. Furthermore, in the lower band the input resistance is equally distributed below and above $50 \Omega$, whereas in the upper band we mostly get $R_{\text {in }}<$ $50 \Omega$. Finally, the $S_{11}$ minimum at $5.77 \mathrm{GHz}$ appears as a result of the small loop traced by the curve, which produces an extra resonance.

The surface current distributions in Figure 10 show concurrent maxima of current density at every point on the PCB. At $2.5 \mathrm{GHz}$ and $5.11 \mathrm{GHz}$ the same comments as in the $\mathrm{K} 2$ and GK2 cases apply. At $5.77 \mathrm{GHz}$, the main arm radiates in an exceptionally uniform manner almost ignoring the presence of the slot, whereas a strong image forms on the ground plane away from the slot.

3.4. Dual-Band PIFA Loaded by the Quadratic Koch Slot. The fourth antenna was inductively loaded by the quadratic Koch-shaped slot of the second kind and second iteration. This particular version of the Koch pre-fractal shape is also known in the literature as the Minkowski sausage. The curve is produced by an iterative function system (IFS) [26-30] as illustrated in Figure 11: an initial straight segment is divided into $n$ equally long, consecutive segments. Then, each one of the $n-2$ middle segments is replaced by a $\Pi$-shaped broken line. The straight segments of the broken line are equal in length to the initial subdivisions. In order for the curve to preserve its self-avoidance, a sequence of proper and inverted $\mathrm{Pi}$ shapes is created. In this way, during the first iteration of the pre-fractal curve, the initial straight segment takes on the shape of a meander line. Thus, we are working with a hybrid meander-Koch curve, and this is where the abbreviation "MK2" for the $i=2$ iteration came from. It is easily proven that, with every new iteration, the updated curve comprises $3 n-4$ straight segments, where $n$ is the number of segments comprising the previous iteration. Therefore, the Haussdorf dimension for $i=+\infty$ is equal to

$$
D_{\mathrm{MK}}=\frac{\ln (3 n-4)}{\ln (n)}
$$

and is maximized when the initial straight segment is divided into $n_{\mathrm{opt}}=\arg \max \left\{D_{\mathrm{MK}}\right\}=4$ subdivisions: $D_{\mathrm{MK}, \max }=$ 1.5. The increased complexity of the MK2 curve leads to two geometrical differences with regard to the previous three antennas: (i) the current covers a longer path as it traverses the initial part of the main arm, thus length $L_{\text {PIFA }}$ turns out to be $8.6-11.7 \%$ shorter when compared to the corresponding lengths of the previous three antennas; (ii) the width of the MK2 slot had to be reduced by a factor of two to facilitate 

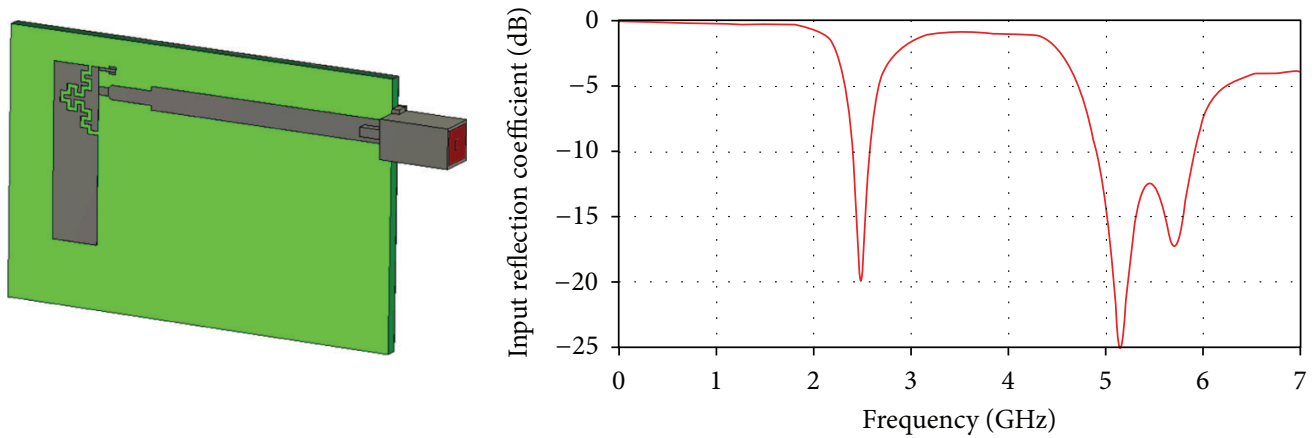

FIGURE 5: The computational model of the dual-band GK2 PIFA in perspective and the magnitude of its input reflection coefficient in the range $\mathrm{DC}-7 \mathrm{GHz}$.

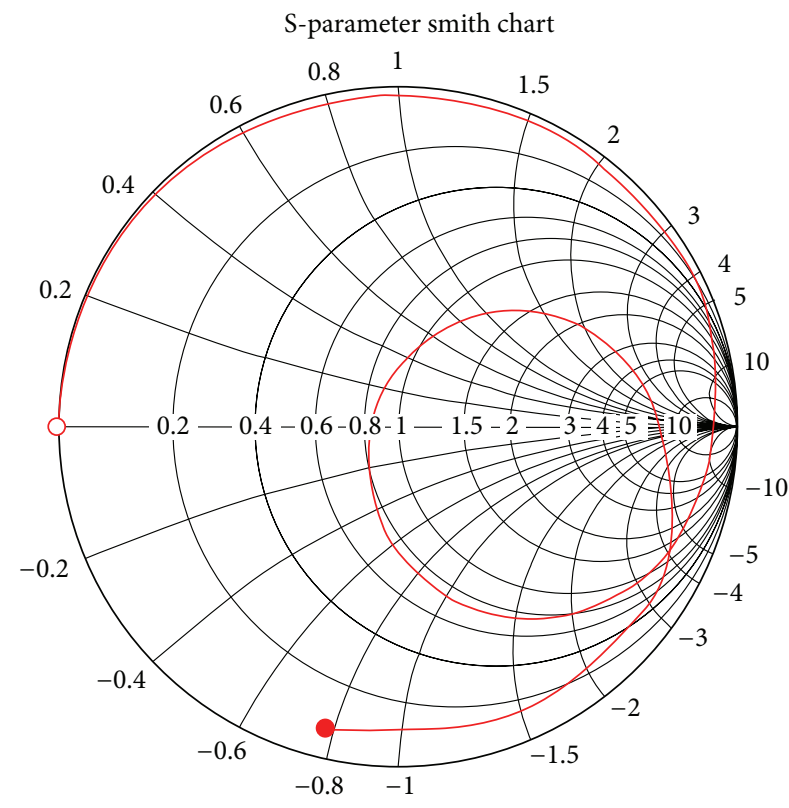

○ $0(-1.875 e-031,-3.063 e-0.15) \mathrm{ohm}$

- $4(3.792,-39.27) \mathrm{ohm}$

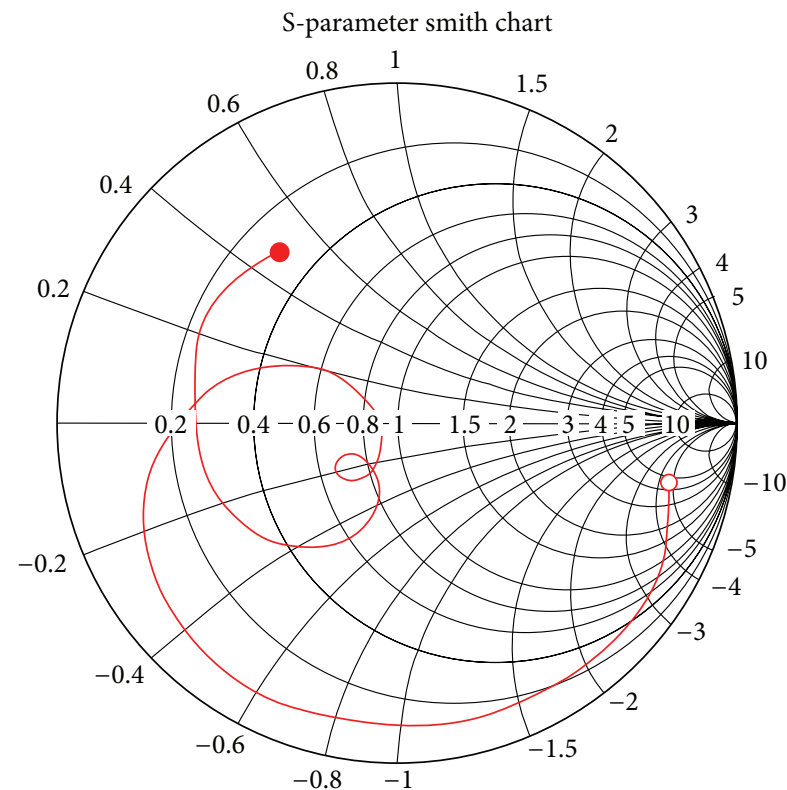

○ $3(232.2,-250.7) \mathrm{ohm}$

- $7(14.93,24.43) \mathrm{ohm}$

(a)

(b)

FIGURE 6: Smith chart of the complex reflection coefficient of the dual-band GK2 PIFA in the overlapping frequency ranges DC-4 GHz and 3-7 GHz.

fabrication. The computational model and the broadband magnitude of the input reflection coefficient are depicted in Figure 12. The antenna resonates at $2.45 \mathrm{GHz}, 5.24 \mathrm{GHz}$, and $5.88 \mathrm{GHz}$. The meander-Koch slot preserves the second $S_{11}$ minimum; however, the matching meets the VSWR criterion only approximately in the $5-6 \mathrm{GHz}$ range. The key numerical results of the K2 PIFA are listed in Table 4.

The Smith charts in Figure 13 indicate that the compensatory dimensions that were chosen for the MK2 antenna produce a frequency response that is well-balanced between inductive and capacitive reactance. Furthermore, in the lower band we get an input resistance $R_{\text {in }}>50 \Omega$, whereas in the upper band we obtain $R_{\mathrm{in}}<50 \Omega$. Finally, in this case, it is the
$S_{11}$ minimum at $5.24 \mathrm{GHz}$ that appears as a result of a small loop traced by the curve.

The surface current distributions in Figure 14 show concurrent maxima of current density at every point on the $\mathrm{PCB}$. At $2.5 \mathrm{GHz}$ the same comments as in the previous three cases apply. At $5.24 \mathrm{GHz}$ and $5.88 \mathrm{GHz}$, the main arm radiates mostly in the vicinity of the slot, whereas a strong image forms on the ground plane along the full extent of the main arm.

3.5. Study of Ground Plane Effect. The effect of the size of the ground plane was studied in the same manner as in $[26,34]$. The most important findings are briefly summarized here. 

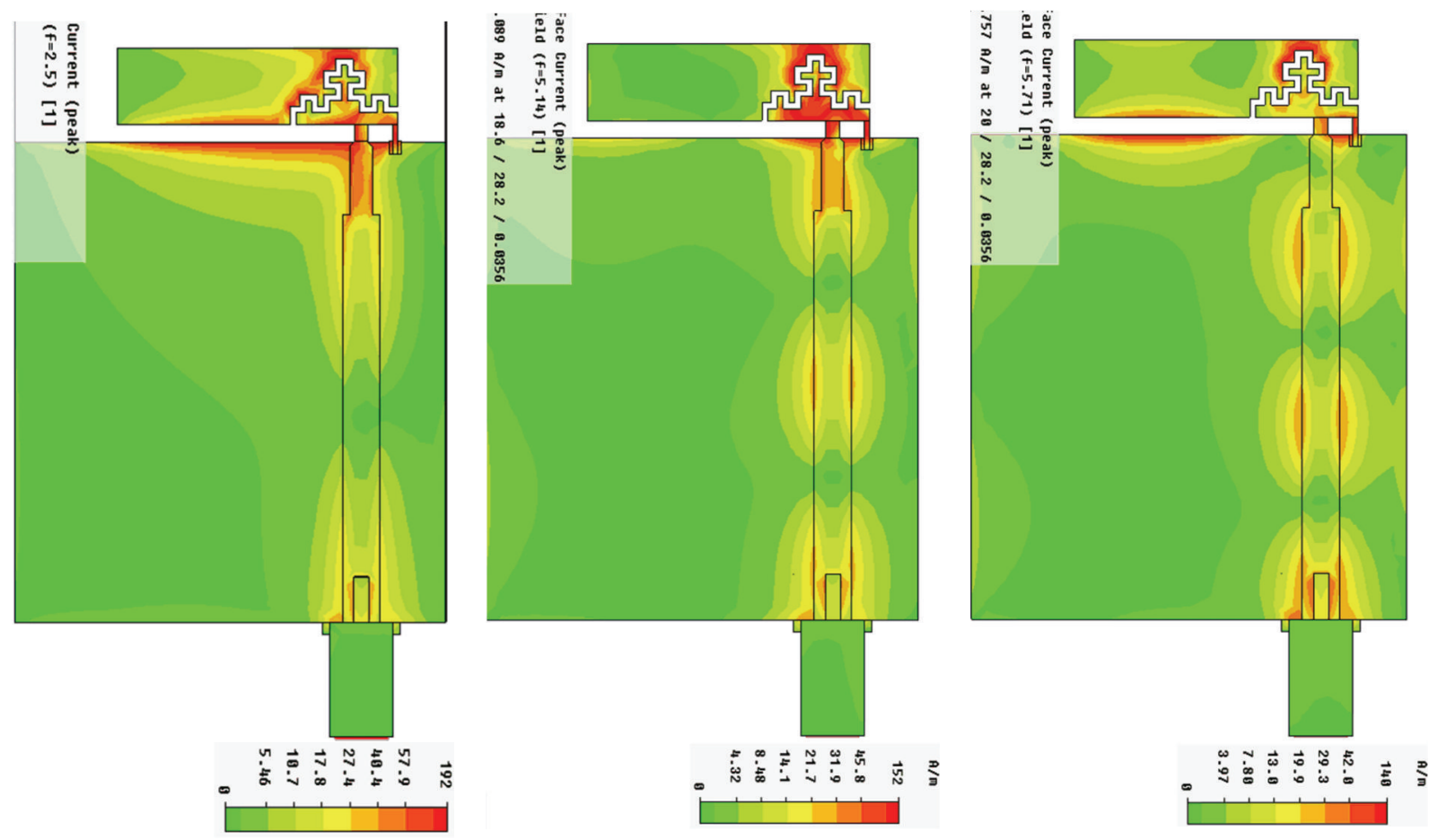

FIGURE 7: Surface current distributions of the dual-band GK2 PIFA at $2.5 \mathrm{GHz}, 5.14 \mathrm{GHz}$ and $5.71 \mathrm{GHz}$.
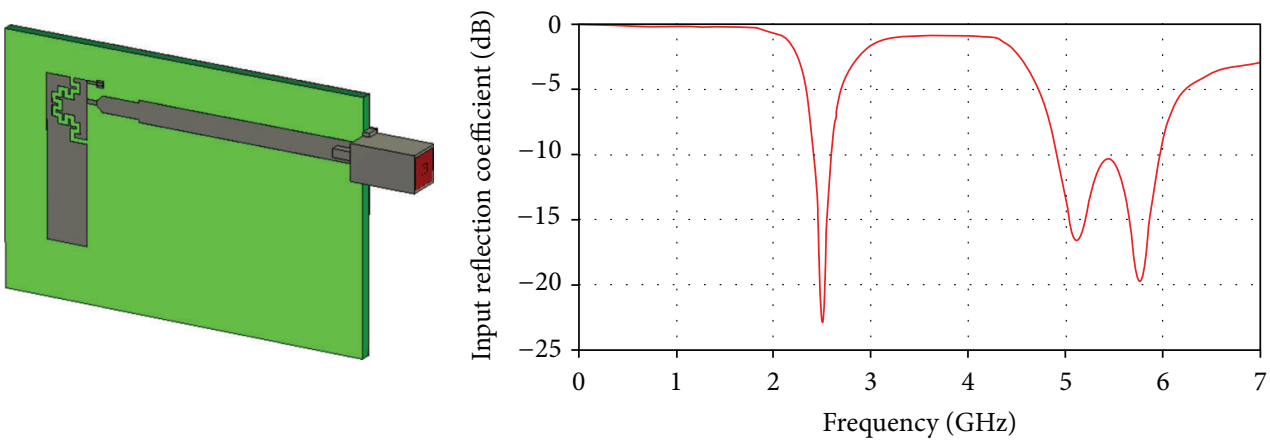

FIGURE 8: The computational model of the dual-band D-GK2 PIFA in perspective and the magnitude of its input reflection coefficient in the range DC-7 GHz.

The width of the ground plane should be at least large enough to accommodate the main arm of the PIFA. Furthermore, small extensions are also required: towards the opencircuited end of the PIFA, the substrate and ground plane should be extended by $8 \leq w_{1} \leq 10 \mathrm{~mm}$. Towards the short-circuited end of the PIFA, the substrate and ground plane should be extended by $3 \leq w_{2} \leq 5 \mathrm{~mm}$. Changes in $w_{1}$ affect both resonances significantly, so this parameter must be chosen carefully. Changes in $w_{2}$ only affect the upper resonance; the lower one is practically insensitive. Note that the values suggested above are frequency-dependent.

The results presented in Sections 3.1-3.4 correspond to the common ground plane length $L_{\text {gnd }}=40 \mathrm{~mm}$. This common length is equal to $\lambda / 3$ at $2.5 \mathrm{GHz}$ and $3 \lambda / 4$ at $5.5 \mathrm{GHz}$. Table 3 lists the ranges of ground length that produce maximum absolute bandwidth and total efficiency for each antenna and frequency band. It seems that optimally compensatory ground length equals $L_{\text {gnd }}^{\prime}=32 \mathrm{~mm}$. This length is equal to $0.27 \lambda$ at $2.5 \mathrm{GHz}$ and $0.59 \lambda$ at $5.5 \mathrm{GHz}$. The $40 \mathrm{~mm}$-long ground plane was chosen because it maximizes the obtainable bandwidth at the lower band.

\section{Discussion}

4.1. Performance of the Four Slotted PIFAs. The results given in Section 3 lead to conclusions and comparisons with prior studies. First, it is important to note that, in the general case, the whole structure is used at both resonances for radiation. Unlike what was suggested in prior studies [9], the current distributions indicate that the slot does not divide the antenna element in two separate radiators, that is, it does not facilitate the creation of two resonant current paths. 


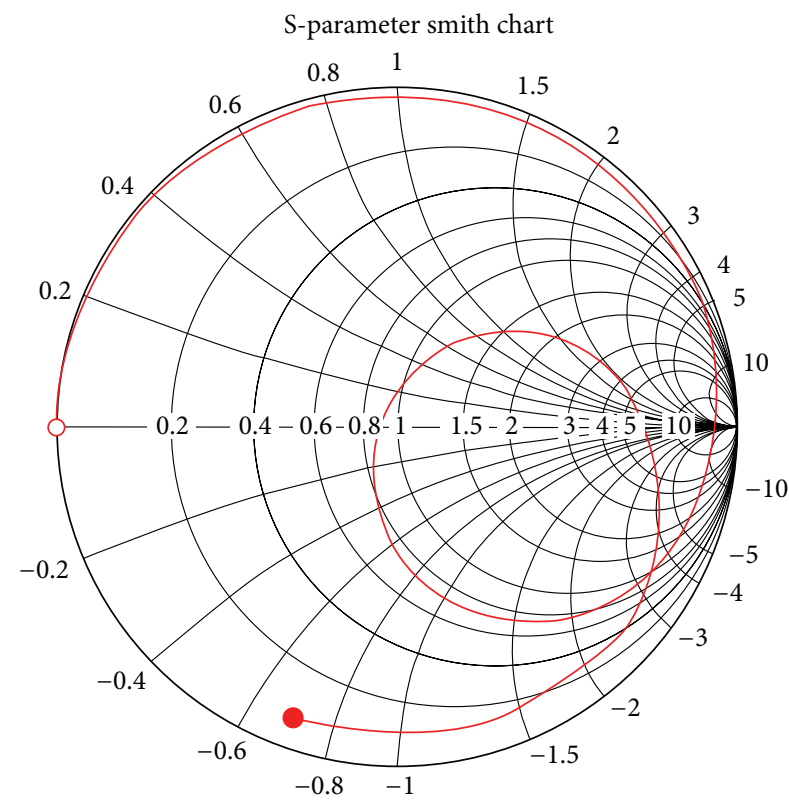

$0(-1.876 e-031,-3.064 e-015) \mathrm{ohm}$

- $4(3.543,-35.16) \mathrm{ohm}$

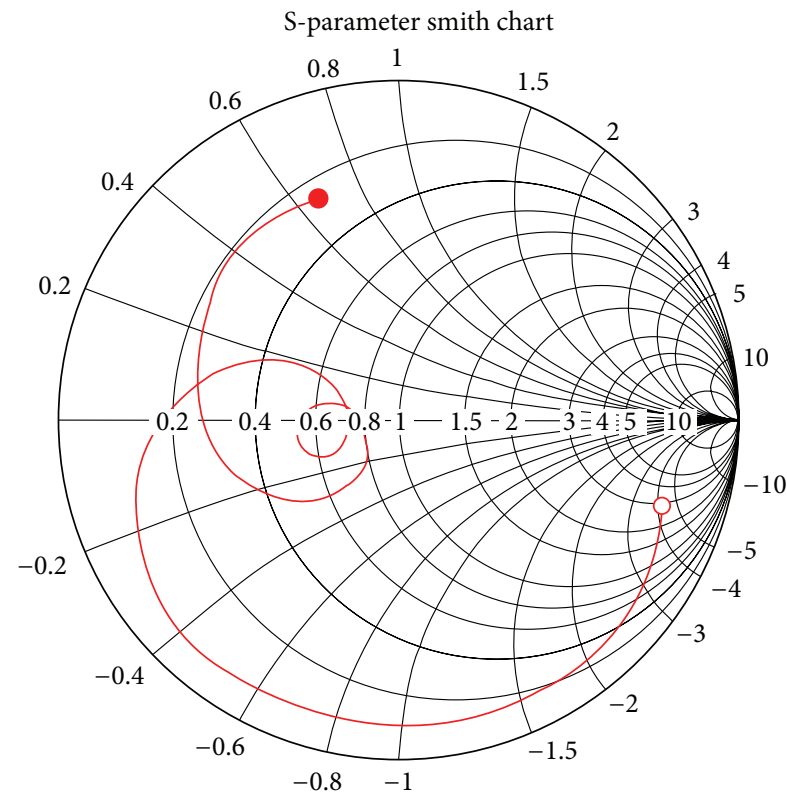

○ $3(146.4,-222.6) \mathrm{ohm}$

- $7(13.29,33.41) \mathrm{ohm}$

(a)

(b)

FIGURE 9: Smith chart of the complex reflection coefficient of the dual-band D-GK2 PIFA in the overlapping frequency ranges DC-4 GHz and $3-7 \mathrm{GHz}$.
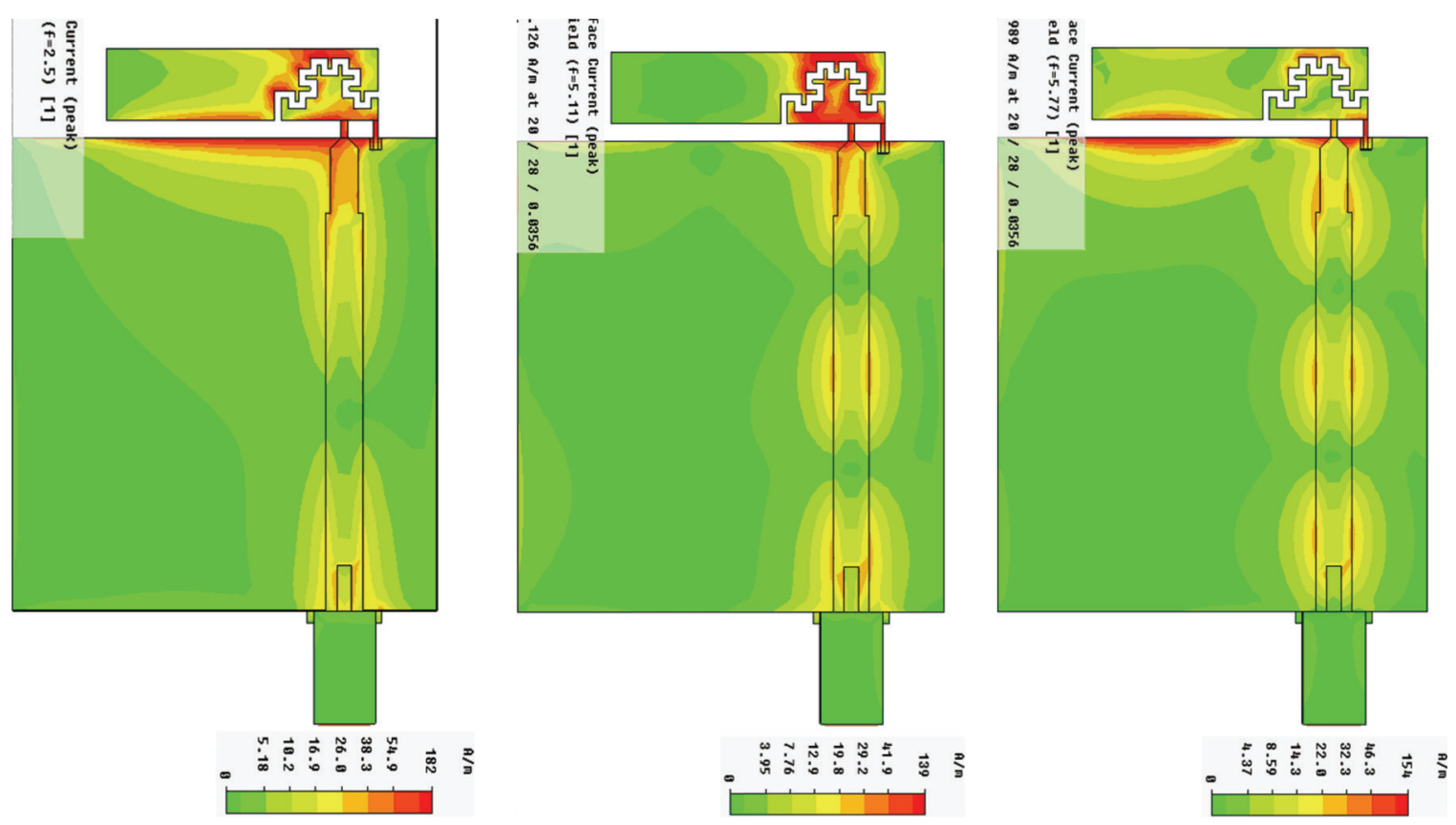

FIGURE 10: Surface current distributions of the dual-band D-GK2 PIFA at $2.5 \mathrm{GHz}, 5.11 \mathrm{GHz}$, and $5.77 \mathrm{GHz}$. 

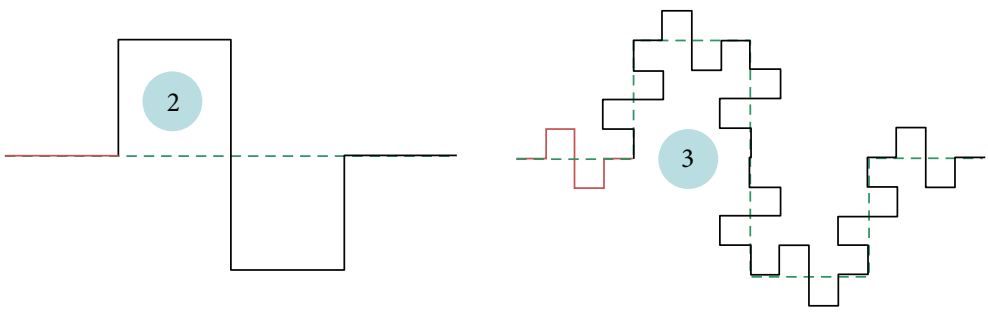

FIGURE 11: The first two iterations, $i=0 \rightarrow 2$, of the hybrid meander-Koch curve for the optimum case of $n=4$ subdivisions.
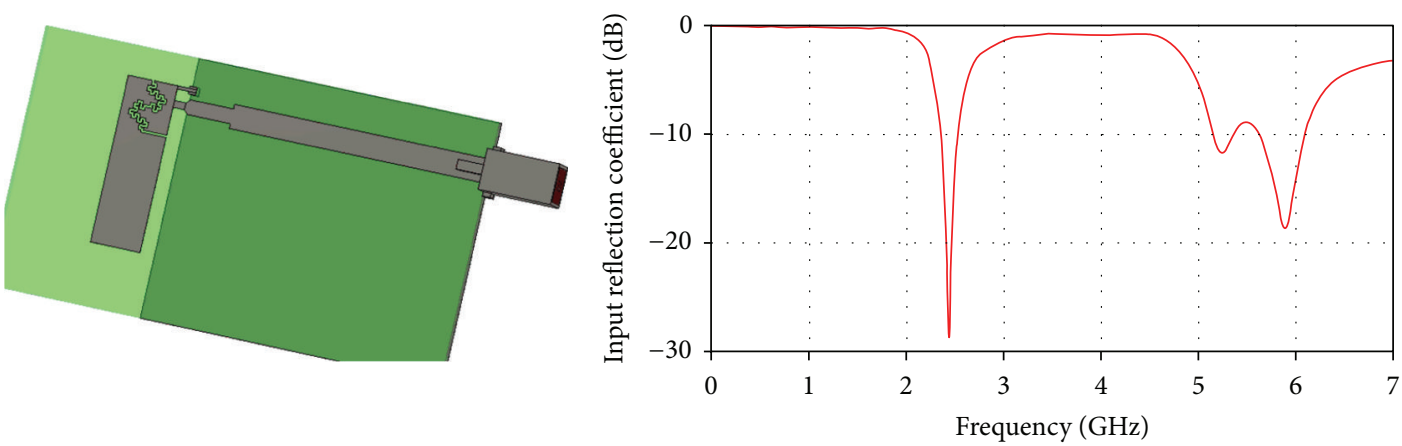

FIGURE 12: The computational model of the dual-band MK2 PIFA in perspective and the magnitude of its input reflection coefficient in the range DC-7 GHz.

TABLE 3: Comparison of well-performing GNDP lengths between the four dual-band printed IFAs in terms of bandwidth and efficiency.

\begin{tabular}{lcccccccc}
\hline & \multicolumn{3}{c}{ K2 } & & GK2 & \multicolumn{2}{c}{ D-GK2 } & MK2 \\
\hline Band $(\mathrm{GHz})$ & $2-3$ & $5-6$ & $2-3$ & $5-6$ & $2-3$ & $5-6$ & $2-3$ & $5-6$ \\
\hline Maximum BW $(\mathrm{GHz})$ & 0.23 & 0.96 & 0.18 & 1.40 & 0.19 & 1.20 & 0.18 & 1.00 \\
at $L_{\text {gnd }}(\mathrm{mm})$ & $36-40$ & 28 & $36-44$ & 28 & $32-44$ & $32-40$ & $32-44$ & 28 \\
\hline Maximum $\overline{n_{\text {total }}}(\%)$ & 88 & $87-88$ & 83 & 91 & 84 & $88-90$ & 84 & $87-88$ \\
at $L_{\text {gnd }}(\mathrm{mm})$ & $32-36$ & $28-32$ & $32-44$ & $28-32$ & $28-44$ & $20-36$ & $28-48$ & 28 \\
\hline
\end{tabular}

TABLE 4: Comparison of electrical performance between the four dual-band printed IFAs in each frequency band.

\begin{tabular}{|c|c|c|c|c|c|c|c|c|}
\hline \multirow[b]{2}{*}{ Band $(\mathrm{GHz})$} & \multicolumn{2}{|c|}{$\mathrm{K} 2$} & \multicolumn{2}{|c|}{ GK2 } & \multicolumn{2}{|c|}{ D-GK2 } & \multicolumn{2}{|c|}{ MK2 } \\
\hline & $2-3$ & $5-6$ & $2-3$ & $5-6$ & $2-3$ & $5-6$ & $2-3$ & $5-6$ \\
\hline$f_{c}(\mathrm{GHz})$ & 2.530 & 5.310 & 2.500 & 5.410 & 2.500 & 5.410 & 2.450 & 5.450 \\
\hline $\mathrm{BW}_{V}(\mathrm{GHz})$ & 0.231 & 0.598 & 0.182 & 1.000 & 0.191 & 1.050 & 0.182 & 0.950 \\
\hline $\mathrm{FBW}_{V}$ & 0.091 & 0.113 & 0.073 & 0.185 & 0.076 & 0.193 & 0.074 & 0.174 \\
\hline Q & 7.3 & 5.9 & 9.1 & 3.6 & 8.7 & 3.4 & 9.0 & 3.8 \\
\hline$k a(\mathrm{rad})$ & 1.60 & 3.35 & 1.56 & 3.38 & 1.57 & 3.41 & 1.50 & 3.20 \\
\hline$\overline{G_{\max }}(\mathrm{dBi})$ & 2.0 & 4.2 & 2.0 & 4.3 & 2.0 & 4.4 & 2.1 & 4.6 \\
\hline$\overline{n_{\mathrm{rad}}}$ & 0.94 & 0.97 & 0.94 & 0.97 & 0.94 & 0.97 & 0.94 & 0.97 \\
\hline$\overline{n_{\text {total }}}$ & 0.87 & 0.82 & 0.83 & 0.86 & 0.84 & 0.87 & 0.83 & 0.84 \\
\hline FOM (dB) & -3.1 & -5.6 & -4.1 & -3.3 & -3.9 & -3.1 & -3.9 & -3.4 \\
\hline$Q_{r}(\mathrm{~dB})$ & +8.9 & +7.8 & +9.9 & +5.7 & +9.7 & +5.4 & +9.8 & +5.9 \\
\hline$Q_{t}(\mathrm{~dB})$ & +9.2 & +8.6 & +10.4 & +6.2 & +10.2 & +5.9 & +10.4 & +6.6 \\
\hline
\end{tabular}




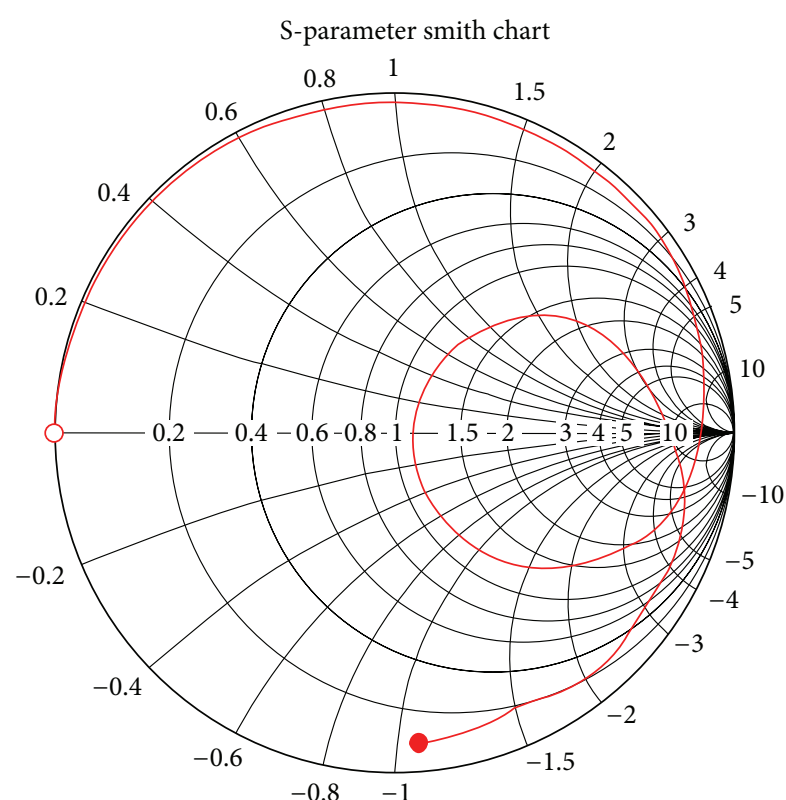

$0(-1.876 e-031,-3.063 e-015) \mathrm{ohm}$

- $4(4.762,-54.23) \mathrm{ohm}$

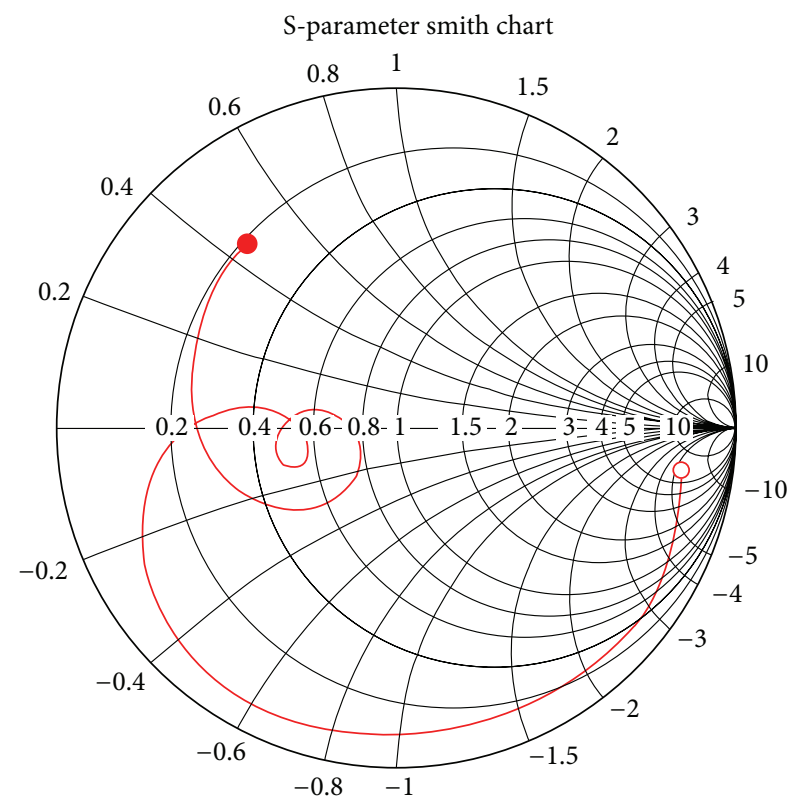

○ $3(330.4,-314)$ ohm

- $7(10.91,22.79) \mathrm{ohm}$

(a)

(b)

FIGURE 13: Smith chart of the complex reflection coefficient of the dual-band MK2 PIFA in the overlapping frequency ranges DC-4 GHz and 3-7 GHz.
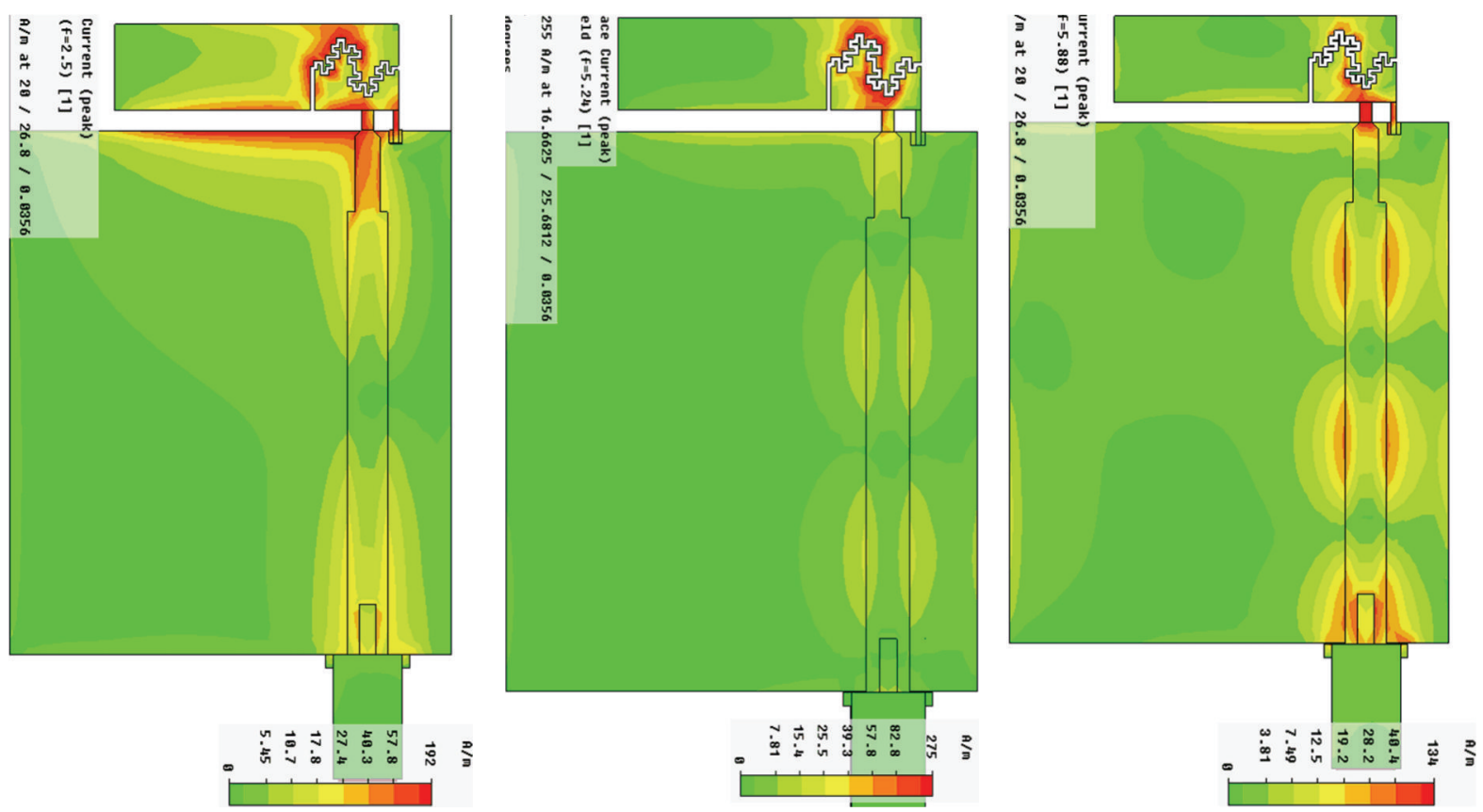

FIGURE 14: Surface current distributions of the dual-band MK2 PIFA at $2.5 \mathrm{GHz}, 5.24 \mathrm{GHz}$, and $5.88 \mathrm{GHz}$.

Rather, it provides the proper reactive loading (or "tuning reactance") for a single-resonant PIFA to become dual band. The computational models corroborate Boyle's theoretical treatment $[5,23,24]$.
Referring to the results tabulated in Table 4, the four dual-band PIFAs meet the specifications that were set for the fractional bandwidth, the electrical size, and the mean total radiation efficiency. The choice of a low-loss substrate leads 


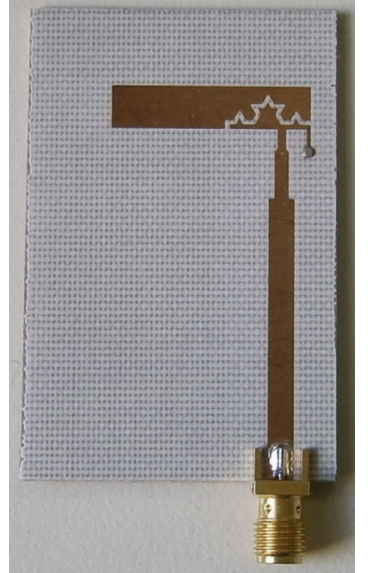

(a)

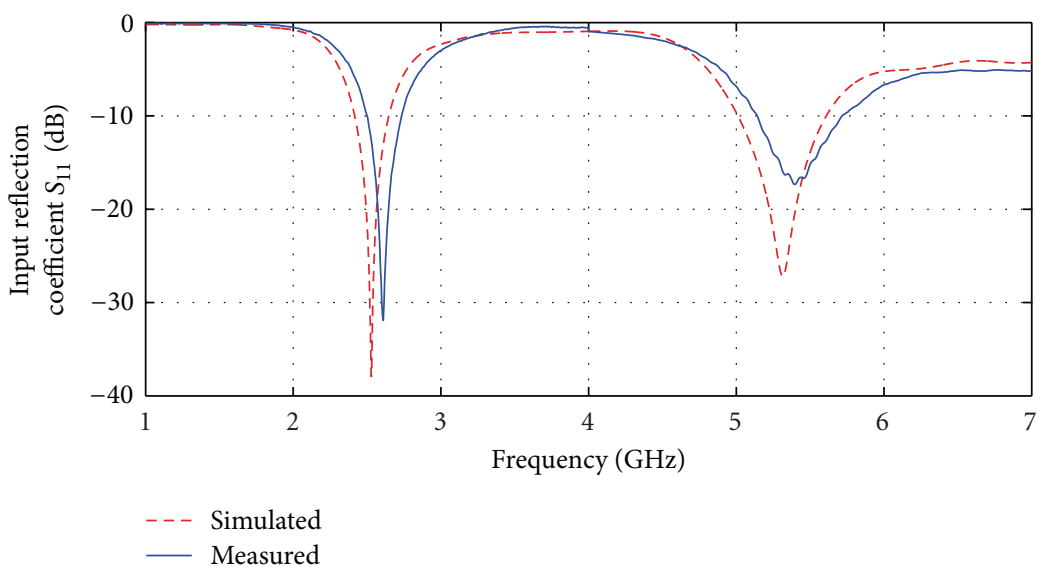

(b)

FIGURE 15: (a) Prototype dual-band PIFA reactively loaded by a K2 slot. (b) Comparison of numerical and experimental magnitudes of the reflection coefficient at the input to the dual-band K2 PIFA.

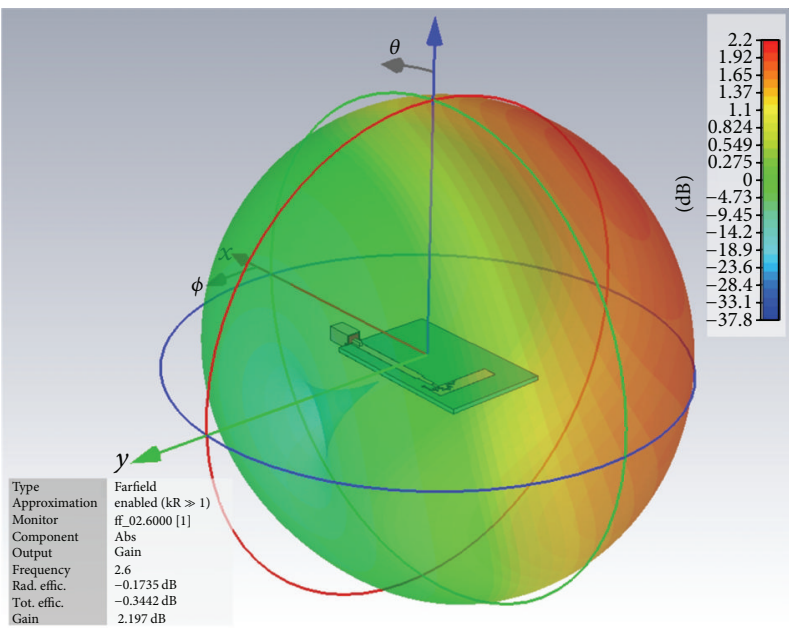

(a)

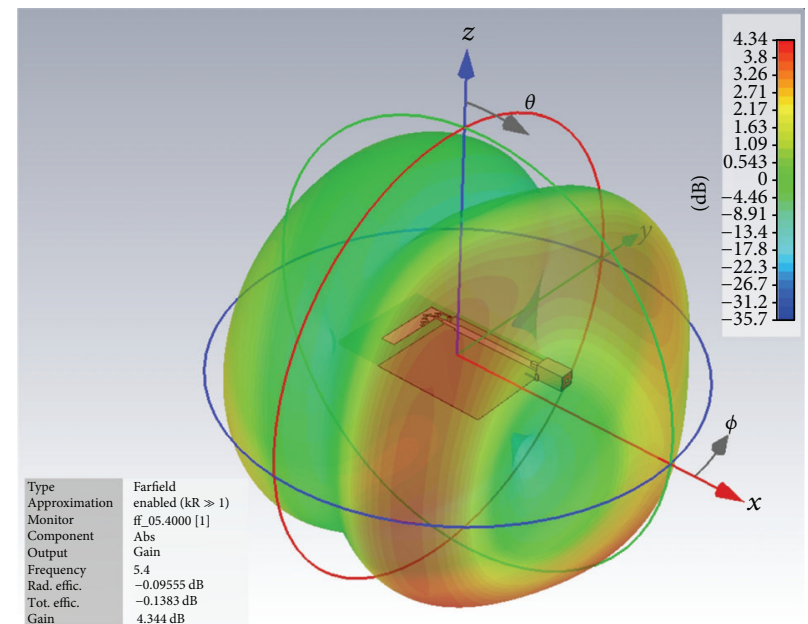

(b)

FIGURE 16: Numerically estimated three-dimensional gain patterns of the K2 PIFA at (a) $2.6 \mathrm{GHz}$ and (b) $5.4 \mathrm{GHz}$. Notice the different amplitude scales (both are given in $\mathrm{dBi}$ ).

to mean radiation efficiencies in the range $94-97 \%$, that is, $-0.3 \leq \overline{n_{\text {rad }}} \leq-0.1 \mathrm{~dB}$. All eight FoM levels take on negative values in decibels. In the lower band, the low FoM values are due to the reduced bandwidth, whereas in the upper band they result from the increased electrical size: in the $5-6 \mathrm{GHz}$ range all four slotted PIFAs are electrically large ( $k a>2 \mathrm{rad})$, a fact that is also attested by the corresponding mean gain values (gain averaged over each band). The expression for the dimensionless figure of merit in decibels is repeated here for convenience,

$$
\begin{aligned}
\mathrm{FOM}= & 10+10 \log \left(\overline{n_{\text {total }}}\right) \\
& +10 \log (\mathrm{FBW})-10 \log (k a) .
\end{aligned}
$$

Karilainen et al. [35] suggested the use of the radiation quality factor, $Q_{r}$, for the comparison of overall performance of antennas featuring different shapes, volumes, and resonant frequencies,

$$
Q_{r}=\frac{Q}{n_{\mathrm{rad}}} .
$$

Taking mismatch losses also into account, the total radiation quality factor, $Q_{t}$, is proposed here in order to evaluate the performance of the antenna system,

$$
Q_{t}=\frac{Q}{\overline{n_{\text {total }}}} .
$$

The values of the dimensionless $Q_{r}$ and $Q_{t}$ in decibels are also tabulated in Table 4 (actually, the former is given as $\left.Q / \overline{n_{\text {rad }}}\right)$. The goal of any given design is to maximize FOM and minimize both $Q_{r}$ and $Q_{t}$. All three metrics paint the same 


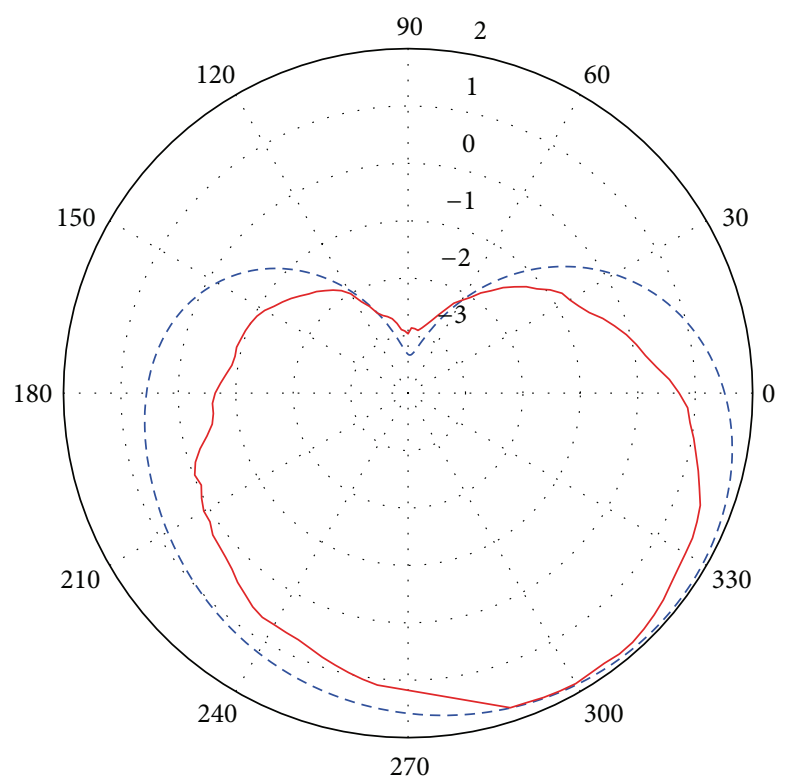

- - Simulated

— Measured

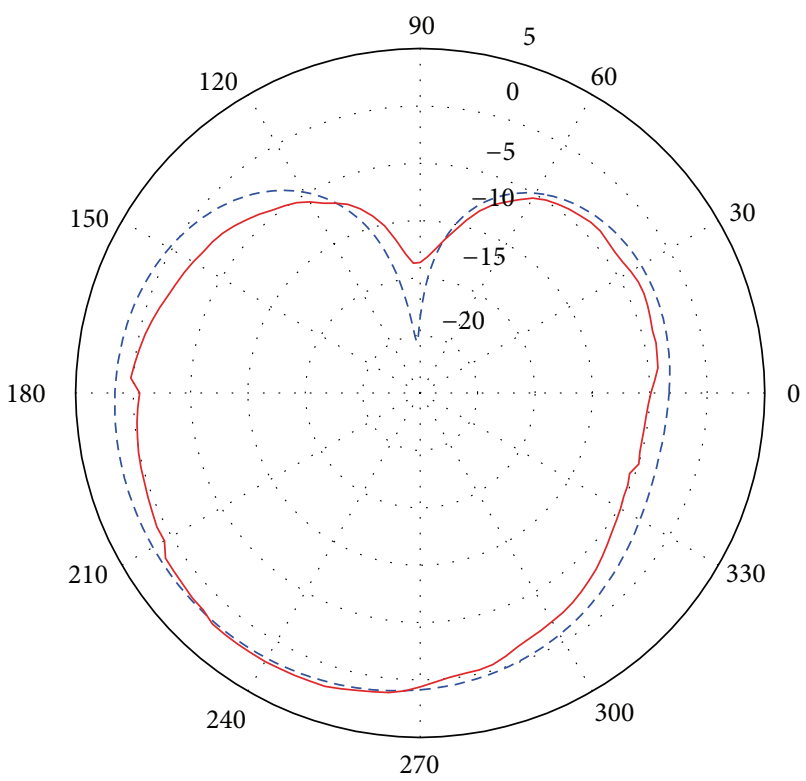

- - Simulated

— Measured

(a)

(b)

FIGURE 17: Comparison of numerical and experimental gain pattern cuts at $2.6 \mathrm{GHz}$ recorded at the principal planes (a) $\phi=0$ and (b) $\theta=\pi / 2$. Notice the different radial scales (both are given in $\mathrm{dBi}$ ).

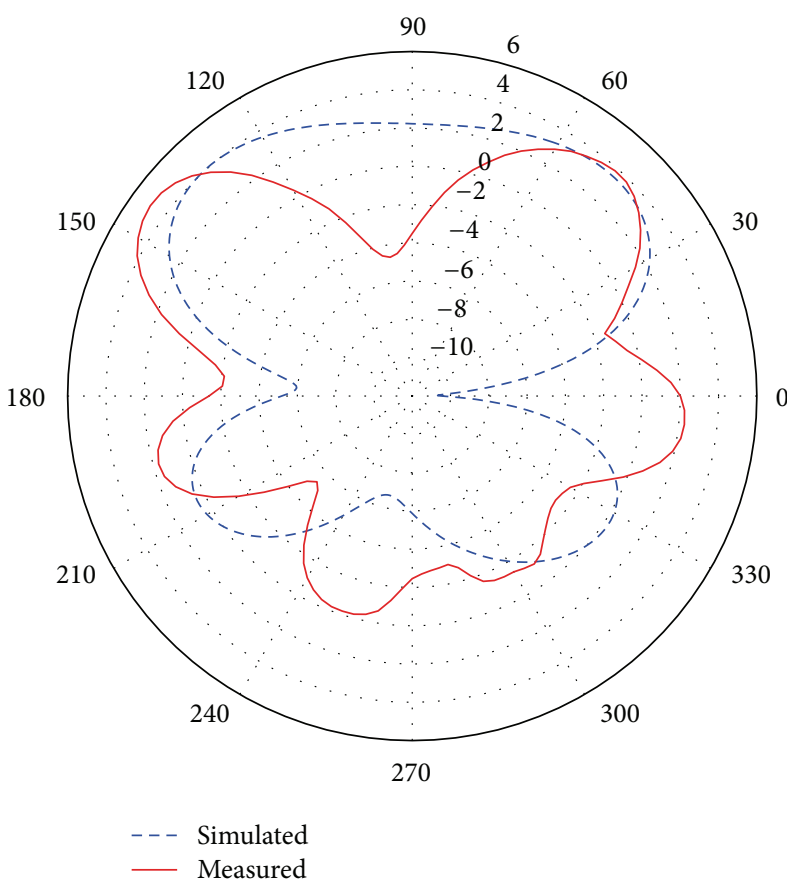

(a)

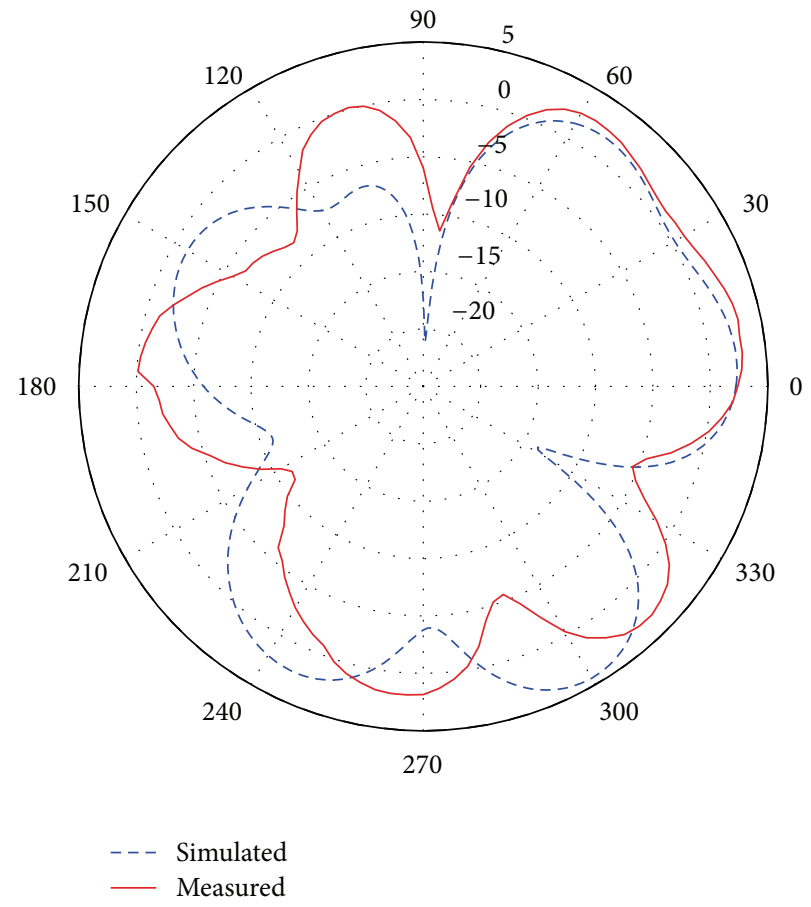

(b)

FIGURE 18: Comparison of numerical and experimental gain pattern cuts at $5.4 \mathrm{GHz}$ recorded at the principal planes (a) $\phi=0$ and (b) $\theta=\pi / 2$. Notice the different radial scales (both are given in $\mathrm{dBi}$ ). 
TABLE 5: Aggregate absolute and fractional bandwidths achieved by the four proposed dual-band PIFAs.

\begin{tabular}{ccccc}
\hline & K2 & GK2 & D-GK2 & MK2 \\
\hline $\mathrm{BW}_{V \ell}+\mathrm{BW}_{V u}(\mathrm{GHz})$ & 0.829 & 1.182 & 1.241 & 1.132 \\
$\mathrm{FBW}_{V \ell}+\mathrm{FBW}_{V u}$ & 0.204 & 0.258 & 0.269 & 0.248 \\
\hline
\end{tabular}

picture with regard to the differential overall performance of the four proposed slotted PIFAs.

Table 5 lists the aggregate bandwidth, absolute and fractional, that was achieved by each PIFA. By comparison of these bandwidth values to the ones given in [26], one concludes that there exist clear indications that the cumulative absolute bandwidth of each antenna is significantly increased. However, this is a superficial conclusion, and it results from the high centre frequency of the upper band, which is more than double the centre frequency of the lower band. The most appropriate indicator of impedance bandwidth is the fractional bandwidth: Table 5 indicates that the aggregate FBW that an antenna, either a single-band or a multi-band one, can yield is strictly limited. Therefore, it is not actually feasible to extract more bandwidth from an antenna by simply triggering more and more resonances. The aggregate FBW of any given antenna is also given, in the sense that it is an intrinsic quality of that particular antenna configuration. The only aspect that the designer can actually influence is how the available fractional bandwidth will be distributed amongst the desired frequency bands.

4.2. Comments on Prior Studies and Performance Comparison. As mentioned in Section 1.1, the only existing study on dualband antennas for wireless sensors is the work of Mendes et al. [10]. They designed a chip-size, multilayered, planar IFA for operation at 2.4 and $5.7 \mathrm{GHz}$. It achieved $50 \mathrm{MHz}$ of bandwidth at $2.4 \mathrm{GHz}(2.1 \%$ fractional $)$ and $170 \mathrm{MHz}$ at $5.7 \mathrm{GHz}(3.0 \%)$. The overall size of the radiator was $6 \mathrm{~mm} \times$ $8.5 \mathrm{~mm} \times 4.5 \mathrm{~mm}$. The circumscribing sphere had a radius $a_{1}=5.7 \mathrm{~mm}$, which corresponds to an electrical size equal to $(k a)_{1}=0.29 \mathrm{rad} \ll 1 \mathrm{rad}$. The definition of electrical size is given in $[26,34]$. Based on data reported in [10], the quality factor of the chip-size planar IFA was estimated at $Q=31.6$. However, the lower bound on $Q$, that is, the wellknown Chu-Harrington-McLean limit, is equal to $Q_{\ell b}=37.6$. One possible explanation for the discrepancy is the effect of the coaxial connector on this electrically small antenna. The antenna operates in dipole mode; hence, there exist radiating currents on the outer sheath of the coaxial connector. If this is indeed the case, then the connector must be included in the circumscribing sphere. The height of the antenna increases from $4.5 \mathrm{~mm}$ to $14.5 \mathrm{~mm}$, and the electrical size becomes $(k a)_{1}^{\prime}=0.45 \mathrm{rad} \ll 1 \mathrm{rad}$. The lower bound on $Q$ becomes $Q_{\ell b}^{\prime}=13.2$, hence no violation occurs.

Moving on to the comparison, Table 6 lists the electrical sizes, fractional bandwidths, and aggregate fractional BWs that were obtained from the literature survey. Note that the estimation of all electrical sizes was based on the assumption that the whole extent of the finite GNDP contributes to the radiation mechanism. The same policy was applied to the four slotted PIFAs (see Table 4). It is also the reason why the work of Wong and Chen [20] was excluded from the comparison; all results reported therein had the antenna mounted on the lid of a laptop, which provided the antenna with an electrically large ground plane. All other structures discussed herein involve strictly finite GNDPs, hence the comparison would not be meaningful.

The aggregate bandwidths listed in Table 6 range between 5-32\%, since the listed PIFAs vary in size from electrically small to electrically very large. All bandwidths were calculated at VSWR $=1.92: 1$, that is, at a reflection level equal to $-10 \mathrm{~dB}$. The aggregate bandwidths of the four slotted PIFAs described herein compare favourably with those reported in prior works. The twelve referenced studies can be divided into three main categories:

(1) PIFA structures that are electrically larger and still produce a smaller aggregate bandwidth compared to the four slotted PIFAs [9, 16-18],

(2) PIFA structures that are electrically smaller and produce a smaller aggregate bandwidth compared to the four slotted PIFAs either because they are smaller, or because they are planar IFAs $[10,11,14,15,21]$, and

(3) PIFA structures that are electrically smaller but manage to produce greater aggregate bandwidths compared to the four slotted PIFAs [12, 13, 19].

The design of the four slotted PIFAs opted for maximization of the bandwidth of the lower resonance, hence the antennas reported herein perform worse compared to design approaches that maximized the upper band or both. Moreover, the electrical size of the slotted PIFAs was influenced not only by the maximization of the lower bandwidth, but also by the need for available real-estate on the PCB to solder the RF and baseband electronics of the wireless sensors. Last but not least, the dual-band technique reported herein also achieves design simplicity and low cost of fabrication.

\section{Measurement Results}

Figure 15(a) depicts a fabricated, dual-band, printed IFA that has been reactively loaded with a Koch-shaped slot of the second iteration (K2). The antenna was fabricated on TRF-45 laminated substrate [31]. Parameter values are in accordance with Table 2.

Measurements were conducted in an fully anechoic chamber (far-field test site), suitable for antenna characterization in the range $0.8-40 \mathrm{GHz}$. The reference antenna was spaced by $4.7 \mathrm{~m}$ from the antenna under test (AUT), that is, by approximately $39 \lambda$ at $2.5 \mathrm{GHz}$. Both AUT and reference antenna were connected through low-loss coaxial cables to the ports of an E8358A vector network analyzer (VNA) [36], which recorded the complex scattering parameters. The settings that were applied to the VNA are listed in Table 7. The measurements were done over two consecutive $3 \mathrm{GHz}$-wide bands. 
5.1. Measured Input Impedance. The result illustrated in Figure 15(b) firstly shows that there is a lower-frequency band exhibiting a resonance at $f_{0, \ell}=2.610 \mathrm{GHz}$, a centre frequency $f_{c, \ell}=2.618 \mathrm{GHz}$, and an operational bandwidth $\mathrm{BW}_{\ell}=0.235 \mathrm{GHz}$. The corresponding fractional bandwidth is $\mathrm{FBW}_{\ell}=0.090$.

At the same time, an upper operating band exists in the 5-6 GHz range. It exhibits a resonance at $f_{0, h}=5.395 \mathrm{GHz}$, a centre frequency $f_{\mathrm{c}, h}=5.428 \mathrm{GHz}$, and an operational bandwidth $\mathrm{BW}_{h}=0.575 \mathrm{GHz}$. The corresponding fractional bandwidth is $\mathrm{FBW}_{\ell}=0.106$.

The results stated above lead to an aggregate absolute bandwidth equal to $\mathrm{BW}_{\ell}+\mathrm{BW}_{h}=0.810 \mathrm{GHz}$. The aggregate fractional bandwidth is equal to $\mathrm{FBW}_{\ell}+\mathrm{FBW}_{h}=0.196$. The results reported in this section compare favourably with the corresponding numerical results listed in Tables 4 and 5 . The relative error in centre frequencies is equal to $+3.5 \%$ and $+2.2 \%$ for the lower and upper band, respectively. Similarly, the differences in absolute bandwidth are $+1.7 \%$ and $-3.8 \%$, whereas the errors in fractional bandwidth read $-1.1 \%$ and $-5.3 \%$. Finally, the deviation in aggregate absolute and fractional bandwidth is $-2.3 \%$ and $-3.9 \%$, respectively.

5.2. Measured Far-Field Patterns. The far-field patterns of the K2 PIFA were measured at 2.6 and $5.4 \mathrm{GHz}$. For the convenience of the reader, the computed three-dimensional patterns of the antenna at these two frequencies are illustrated in Figure 16. The antenna lies on the $x y$-plane, as shown by the coordinate system in Figure 16. The surface currents depicted in Figures 4, 7, 10, and 14 indicate that the radiating currents feature strong $x$ - and $y$-components. The orthogonal currents are phased randomly. Thus, as anticipated, the four printed IFAs are elliptically polarized in the general case. Therefore, the gain amplitudes given in Figure 16 correspond to the vectorial sum of the $\theta$ - and $\phi$-components of the $\mathbf{E}$ field.

The far-field measurements discussed here report on the results that were harvested from the $\phi=0$ and $\theta=$ $\pi / 2$ principal planes. The reference antenna was a linearly polarized, standard-gain horn; hence, the $\theta$ (vertical) and $\phi$ (horizontal) components of the far field were measured separately. The results are grouped in Figures 17 and 18; the gain amplitudes again correspond to the vectorial sum of the $\theta$ - and $\phi$-components of the E-field.

At $2.6 \mathrm{GHz}$ there is good agreement between computed and measured patterns, especially in the $\theta=\pi / 2$ cut. The directions of maxima and minima have been predicted with considerable accuracy. In the $\phi=0$ cut, the measured maximum IEEE gain lags the computed one by $0.1 \mathrm{~dB}(1.9 \mathrm{dBi}$ versus $2.0 \mathrm{dBi}$ ). On the other hand, in the $\theta=\pi / 2$ cut, the measured maximum gain exceeds the computed one by $0.2 \mathrm{~dB}(2.1 \mathrm{dBi}$ versus $1.9 \mathrm{dBi})$. A deviation on the order of $\pm 0.2 \mathrm{~dB}$ lies within the measurement uncertainty of the VNA [36], which can be estimated by the instrument settings listed in Table 7.

At $5.4 \mathrm{GHz}$ the agreement between computed and measured patterns becomes worse, particularly in the $\phi=0$ cut. Significant null filling is observed, together with angular shifting of the side lobes. The deviant behaviour is attributed to radiation from the feed cable, which amplifies the $\theta$ component, and to scattering from the mounting pole and tripod; according to the $2 D^{2} / \lambda$ rule, the reactive and radiating near field of the antenna occupies twice the space when firing in the upper band. Nevertheless, in the $\phi=0$ cut, the measured maximum IEEE gain exceeds the computed one by $0.9 \mathrm{~dB}$ ( $4.9 \mathrm{dBi}$ versus $4.0 \mathrm{dBi}$ ). On the other hand, in the $\theta=\pi / 2$ cut, the measured maximum gain lags the computed one by $1.2 \mathrm{~dB}$ (3.2 $\mathrm{dBi}$ versus $4.4 \mathrm{dBi})$.

\section{Conclusion}

Dual-band communication can play a significant role in current and future wireless sensor networks. Given the scarcity of relevant proper radiators, this paper described how printed antennas can be efficiently designed as simple, compact, device-integrated, dual-band antennas. During the evolution of modern antennas, the planar IFA inherited the properties of the microstrip "patch" antenna, while the printed IFA inherited those of the printed monopole. Printed monopoles are well-known wideband radiators; patch antennas are not. Reactive tuning of printed IFAs was applied in the form of four slotted configurations, in order to exert as much aggregate bandwidth as possible for use in two well-known unlicensed bands.

Electrical performance was characterized through numerous computed results. Slot loading showed a potential to increase the impedance bandwidth compared to prior implementations in terms of achievable $\mathrm{FBW}_{V}$. The study of SCDs revealed that most of the area of the element is used for radiation at both resonances. In radiation terms, the antennas provided satisfactory gains and high efficiencies $(\geq 82 \%)$. A simple figure of merit was used to compare the performance of the three PIFAs head to head. The final comparison displayed in an emphatic way that modern antenna design is an art of compromise.

By exploring the potential for operation at $2.5 \mathrm{GHz}$ and $5.5 \mathrm{GHz}$, it was discovered that a simple change in slot geometry can almost double the achievable operational bandwidth. Thus, the proposed antennas not only serve the $5.15-5.35 \mathrm{GHz}$ U-NII band, but also the $5.725-5.875 \mathrm{GHz}$ ISM band. The proposed dual-band structures exhibited at the lower band an electrical size less or equal to that of the half-wavelength dipole. Nonetheless, these antennas can indeed be considered compact, even though they are not electrically small per se, since their dual-band capability enables them to do the job of two and even three separate antennas. Meanwhile, an educated choice of substrate can enable the combination of ease of fabrication and high radiation efficiency even at midC-band.

Yet another important design element that this study of dual-band antennas highlighted is that the aggregate fractional bandwidth of a PIFA is bounded within certain limits, and by degenerating higher-order radiating modes the designer merely re-distributes whatever bandwidth is available by the antenna itself to the desired frequency bands. 
TABLE 6: Electrical sizes, fractional bandwidths, and aggregate fractional bandwidths from the literature survey.

\begin{tabular}{|c|c|c|c|c|c|}
\hline \multirow{2}{*}{ Reference work } & \multicolumn{2}{|c|}{ Lower band } & \multicolumn{2}{|c|}{ Upper band } & \multirow[b]{2}{*}{ Aggr. FBW } \\
\hline & $k a(\mathrm{rad})$ & FBW & $k a(\mathrm{rad})$ & FBW & \\
\hline Angelopoulos et al. [9] & 3.03 & 0.086 & 6.47 & 0.063 & 0.149 \\
\hline Mendes et al. [10] & 0.45 & 0.021 & 1.07 & 0.030 & 0.051 \\
\hline Moon et al. [11] & 0.79 & 0.041 & 1.90 & 0.145 & 0.186 \\
\hline Cho et al. [12] & 0.60 & 0.057 & 1.39 & 0.261 & 0.318 \\
\hline Nakano et al. [13] & 1.11 & 0.041 & 2.65 & 0.282 & 0.323 \\
\hline Azad and Ali [14] & 1.13 & 0.070 & 2.36 & 0.033 & 0.103 \\
\hline Cho et al. [15] & 1.33 & 0.045 & 3.02 & 0.162 & 0.207 \\
\hline Wang et al. [16] (spiralled tail) & 1.97 & 0.057 & 4.22 & 0.144 & 0.201 \\
\hline Wang et al. [16] (coupling element) & 1.94 & 0.098 & 4.15 & 0.128 & 0.226 \\
\hline Michailidis et al. [17] & 1.72 & 0.095 & 3.59 & 0.137 & 0.232 \\
\hline Chan et al. [18] & 2.09 & 0.109 & 2.83 & 0.036 & 0.145 \\
\hline Yu and Choi [19] & 1.14 & 0.187 & 2.51 & 0.153 & 0.340 \\
\hline Li et al. [21] (IFA mode) & 1.16 & 0.085 & 3.04 & 0.078 & 0.163 \\
\hline
\end{tabular}

TABLE 7: Settings applied to the network analyzer during the measurement of the K2 PIFA.

\begin{tabular}{lc}
\hline \multicolumn{2}{c}{ Network analyzer settings } \\
\hline Span (Cal + Meas) & $1-4 \mathrm{GHz}$ and $4-7 \mathrm{GHz}$ \\
IF bandwidth & $5 \mathrm{kHz}$ \\
Number of points & $601(\Delta f=5 \mathrm{MHz})$ \\
Sweep time & $128 \mathrm{msec}($ manual $)$ \\
Averaging (full span) & $32(15 \mathrm{~dB})$ \\
\hline
\end{tabular}

\section{Acknowledgments}

C. Kakoyiannis thanks Dr. T. Zervos and Dr. A. Alexandridis, both with the Wireless Communications Laboratory, Institute of Informatics and Telecommunications, NCSR "Demokritos," Athens, Greece, for assisting with the antenna measurements that were conducted in the anechoic facility of the institute. The authors wish to acknowledge Taconic Advanced Dielectric Division (Mullingar, Ireland, and Cheonan, South Korea) for donating the TRF-45 laminated substrate, which was used for design and fabrication of all antennas described herein. This work is part of the "MAGELLAN" research project, implemented within the framework of the “THALIS” Research Programme (NSRF 2007-2013), and cofinanced by the Greek Ministry of Education and Religious Affairs, Culture and Sports and the European Union.

\section{References}

[1] P. S. Hall, E. Lee, and C. T. P. Song, "Planar inverted-F antennas," in Printed Antennas for Wireless Communications, R. Waterhouse, Ed., chapter 7, pp. 197-228, John Wiley \& Sons, Chichester, UK, 2007.

[2] B. S. Collins, "Handset antennas," in Antennas for Portable Devices, Z. N. Chen, Ed., chapter 2, John Wiley \& Sons, Chichester, UK, 2007.

[3] Y. C. Vardaxoglou and J. R. James, "Mobile handset antennas," in Antenna Engineering Handbook, J. L. Volakis, Ed., chapter 36, McGraw-Hill, New York, NY, USA, 4th edition, 2007.
[4] Z. Ying, M. Ohtsuka, Y. Nishioka, and K. Fujimoto, "Antennas for mobile terminals," in Mobile Antenna Systems Handbook, K. Fujimoto, Ed., chapter 5, pp. 213-320, Artech House, Norwood, Mass, USA, 3rd edition, 2008.

[5] K. Boyle, "Multiband multisystem antennas in handsets", in Multiband Integrated Antennas for $4 G$ Terminals, D. A. SánchezHernández, Ed., chapter 2, pp. 33-52, Artech House, Norwood, Mass, USA, 2008.

[6] M. Martínez-Vázquez, E. Antonino-Daviú, and M. FabedoCabrés, "Miniaturized integrated multiband antennas," in Multiband Integrated Antennas for $4 G$ Terminals, D. A. SánchezHernández, Ed., chapter 5, pp. 151-186, Artech House, Norwood, Mass, USA, 2008.

[7] Y. Rahmat-Samii, J. Guterman, A. A. Moreira, and C. Peixeiro, "Integrated antennas for wireless personal communications," in Modern Antenna Handbook, C. A. Balanis, Ed., chapter 22, pp. 1079-1142, John Wiley \& Sons, Hoboken, NJ, USA, 2008.

[8] K. Fujimoto, "Antennas for mobile communications," in Modern Antenna Handbook, C. A. Balanis, Ed., chapter 23, pp. 11431228, John Wiley \& Sons, Hoboken, NJ, USA, 2008.

[9] E. S. Angelopoulos, A. I. Kostaridis, and D. I. Kaklamani, "A novel dual-band f-inverted antenna printed on a PCMCIA card," Microwave and Optical Technology Letters, vol. 42, no. 2, pp. 153-156, 2004.

[10] P. M. Mendes, A. Bartek, J. N. Burghartz, and J. H. Correia, "Novel very small dual-band chip-size antenna for wireless sensor networks," in Proceedings of the IEEE Radio and Wireless Conference (RAWCON '04), pp. 419-422, Atlanta, Ga, USA, September 2004.

[11] J. I. Moon, D. U. Sim, and S. O. Park, "Compact PIFA for 2.4/5 GHz dual ISM-band applications," Electronics Letters, vol. 40, no. 14, pp. 844-846, 2004.

[12] Y. J. Cho, S. H. Hwang, and S. O. Park, "Printed antenna with folded non-uniform meander line for $2.4 / 5 \mathrm{GHz}$ WLAN bands," Electronics Letters, vol. 41, no. 14, pp. 786-788, 2005.

[13] H. Nakano, Y. Sato, H. Mimaki, and J. Yamauchi, "An inverted FL antenna for dual-frequency operation," IEEE Transactions on Antennas and Propagation, vol. 53, no. 8, pp. 2417-2421, 2005.

[14] M. Z. Azad and M. Ali, "A miniaturized hilbert PIFA for dualband mobile wireless applications," IEEE Antennas and Wireless Propagation Letters, vol. 4, no. 1, pp. 59-62, 2005. 
[15] Y. J. Cho, Y. S. Shin, and S. O. Park, "Internal PIFA for 2.4/5 GHz WLAN applications," Electronics Letters, vol. 42, no. 1, pp. 8-9, 2006.

[16] Y. S. Wang, M. C. Lee, and S. J. Chung, "Two PIFA-related miniaturized dual-band antennas," IEEE Transactions on Antennas and Propagation, vol. 55, no. 3, pp. 805-811, 2007.

[17] E. Michailidis, C. C. Tsimenidis, and E. G. Chester, "Parametric study of dual and wide band PIFA," in Proceedings of the IET Seminar on Wideband Multiband Antennas and Arrays for Defence or Civil Applications, pp. 145-159, London, UK, March 2008.

[18] P. W. Chan, H. Wong, and E. K. N. Yung, "Dual-band printed inverted-F antenna for DCS, 2.4 GHZ WLAN applications," in Proceedings of the Loughborough Antennas and Propagation Conference (LAPC '08), pp. 185-188, Loughborough, UK, March 2008.

[19] Y. Yu and J. Choi, "Compact internal inverted-F antenna for USB dongle applications," Electronics Letters, vol. 45, no. 2, pp. 92-94, 2009.

[20] K. L. Wong and W. J. Chen, "Small-size microstrip-coupled printed PIFA for 2.4/5.2/5.8 GHz WLAN operation in the laptop computer," Microwave and Optical Technology Letters, vol. 51, no. 9, pp. 2072-2076, 2009.

[21] Y. Li, Z. Zhang, J. Zheng, Z. Feng, and M. F. Iskander, "A compact hepta-band loop-inverted $\mathrm{F}$ reconfigurable antenna for mobile phone," IEEE Transactions on Antennas and Propagation, vol. 60, no. 1, pp. 389-392, 2012.

[22] N. Cho, J. Bae, and H. J. Yoo, "A $10.8 \mathrm{~mW}$ body channel communication/MICS dual-band transceiver for a unified body sensor network controller," IEEE Journal of Solid-State Circuits, vol. 44, no. 12, pp. 3459-3468, 2009.

[23] K. Boyle, Antennas for multi-band RF front-end modules [Ph.D. thesis], Delft University of Technology, Delft, The Netherlands, 2004.

[24] K. R. Boyle and L. P. Ligthart, "Radiating and balanced mode analysis of PIFA antennas," IEEE Transactions on Antennas and Propagation, vol. 54, no. 1, pp. 231-237, 2006.

[25] Z. D. Liu, P. S. Hall, and D. Wake, "Dual-frequency planar inverted-F antenna," IEEE Transactions on Antennas and Propagation, vol. 45, no. 10, pp. 1451-1458, 1997.

[26] C. G. Kakoyiannis, A. Kyrligkitsi, and P. Constantinou, "Bandwidth enhancement, radiation properties and grounddependent response of slotted antennas integrated into wireless sensors," IET Microwaves, Antennas and Propagation, vol. 4, no. 5, pp. 609-628, 2010.

[27] C. Puente, J. Romeu, and A. Cardama, "Fractal-shaped antennas," in Frontiers in Electromagnetics, D. Werner and R. Mittra, Eds., chapter 2, pp. 48-93, IEEE Press, New York, NY, USA, 2000.

[28] J. P. Gianvittorio and Y. Rahmat-Samii, "Fractal antennas: a novel antenna miniaturization technique, and applications," IEEE Antennas and Propagation Magazine, vol. 44, no. 1, pp. 20 36, 2002.

[29] D. H. Werner and S. Ganguly, "An overview of fractal antenna engineering research," IEEE Antennas and Propagation Magazine, vol. 45, no. 1, pp. 38-57, 2003.

[30] W. J. Krzystofik, "Printed multiband fractal antennas," in Multiband Integrated Antennas for $4 G$ TermInals, D. A. SánchezHernández, Ed., chapter 4, pp. 95-150, Artech House, Norwood, Mass, USA, 2008.

[31] Advanced PCB Materials Product Selection Guide, Taconic, Petersburgh, NY, USA, 2010.
[32] T. Weiland, M. Timm, and I. Munteanu, "A practical guide to 3-D simulation," IEEE Microwave Magazine, vol. 9, no. 6, pp. 62-75, 2008.

[33] I. Munteanu, M. Timm, and T. Weiland, "It's about time," IEEE Microwave Magazine, vol. 11, no. 2, pp. 60-69, 2010.

[34] C. G. Kakoyiannis and P. Constantinou, "Radiation properties and ground-dependent response of compact printed sinusoidal antennas and arrays," IET Microwaves, Antennas and Propagation, vol. 4, no. 5, pp. 629-642, 2010.

[35] A. O. Karilainen, P. M. T. Ikonen, C. R. Simovski et al., "Experimental studies on antenna miniaturisation using magnetodielectric and dielectric materials," IET Microwaves, Antennas and Propagation, vol. 5, no. 4, pp. 495-502, 2011.

[36] Agilent PNA Series RF Network Analyzers E8356/7/8A, E8801/2/3A, N3381/2/3A Data Sheet, Agilent Technologies Inc., Santa Rosa, Calif, USA, 2006. 

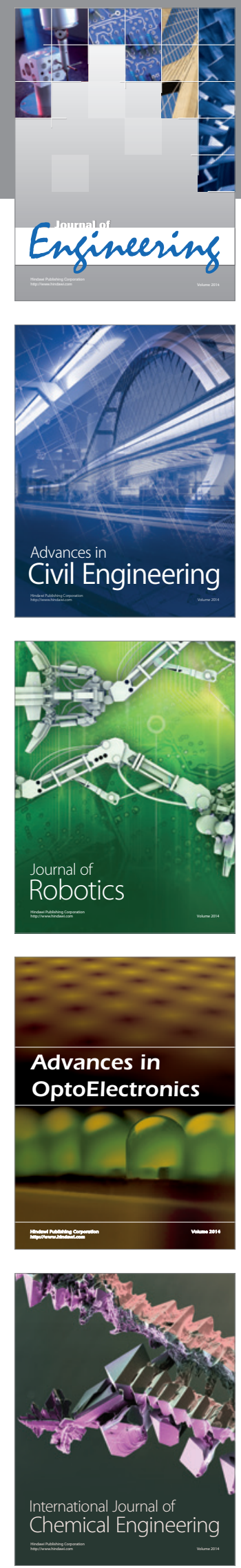

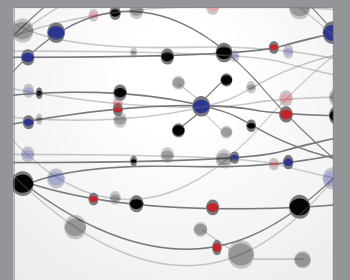

The Scientific World Journal
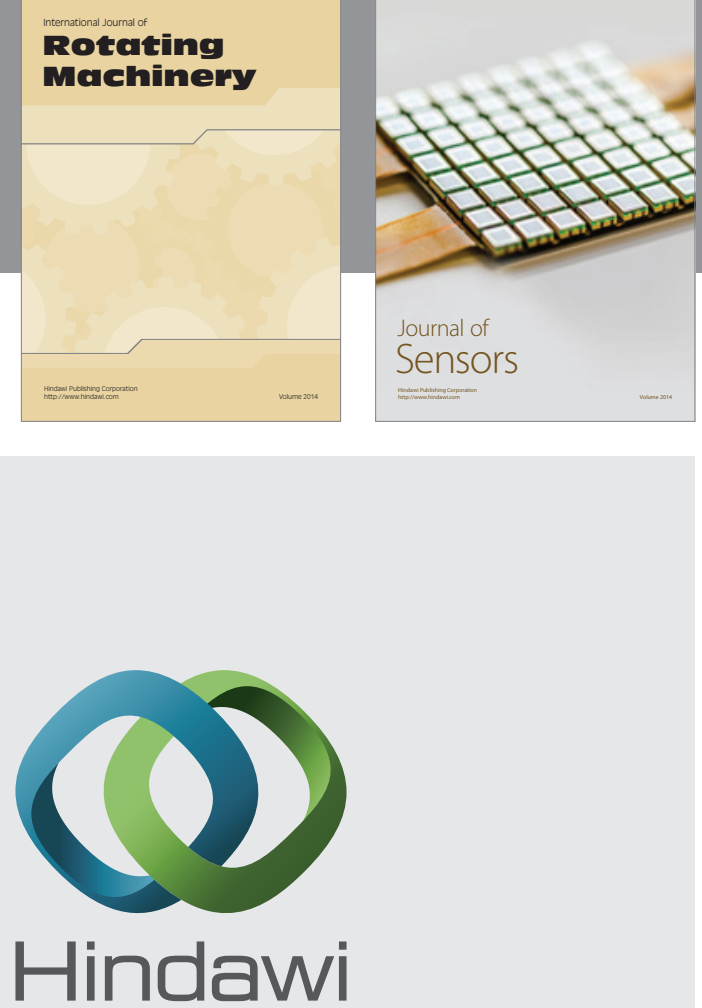

Submit your manuscripts at http://www.hindawi.com
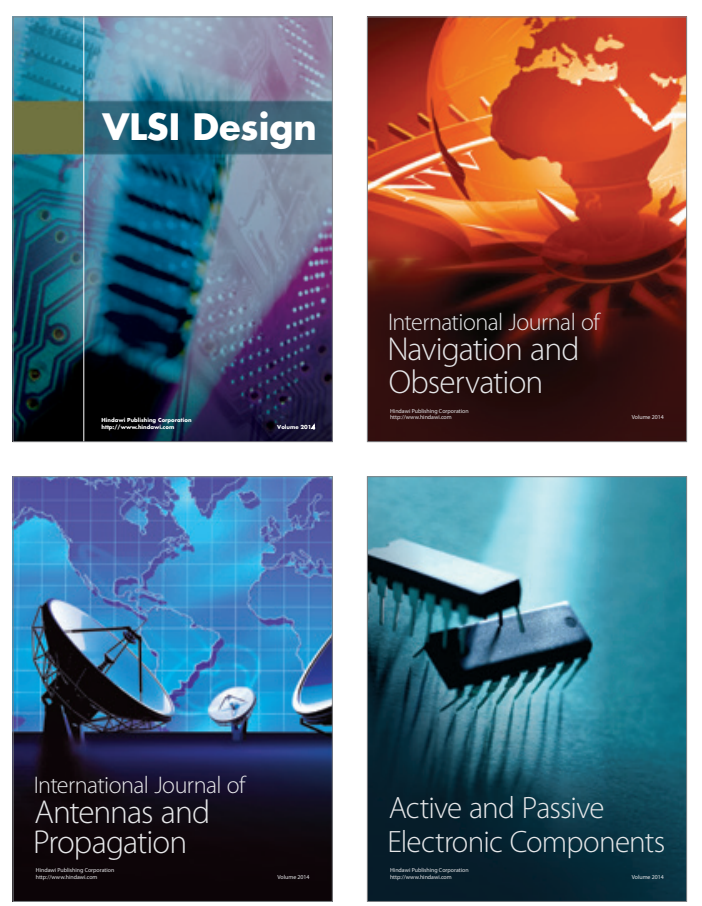
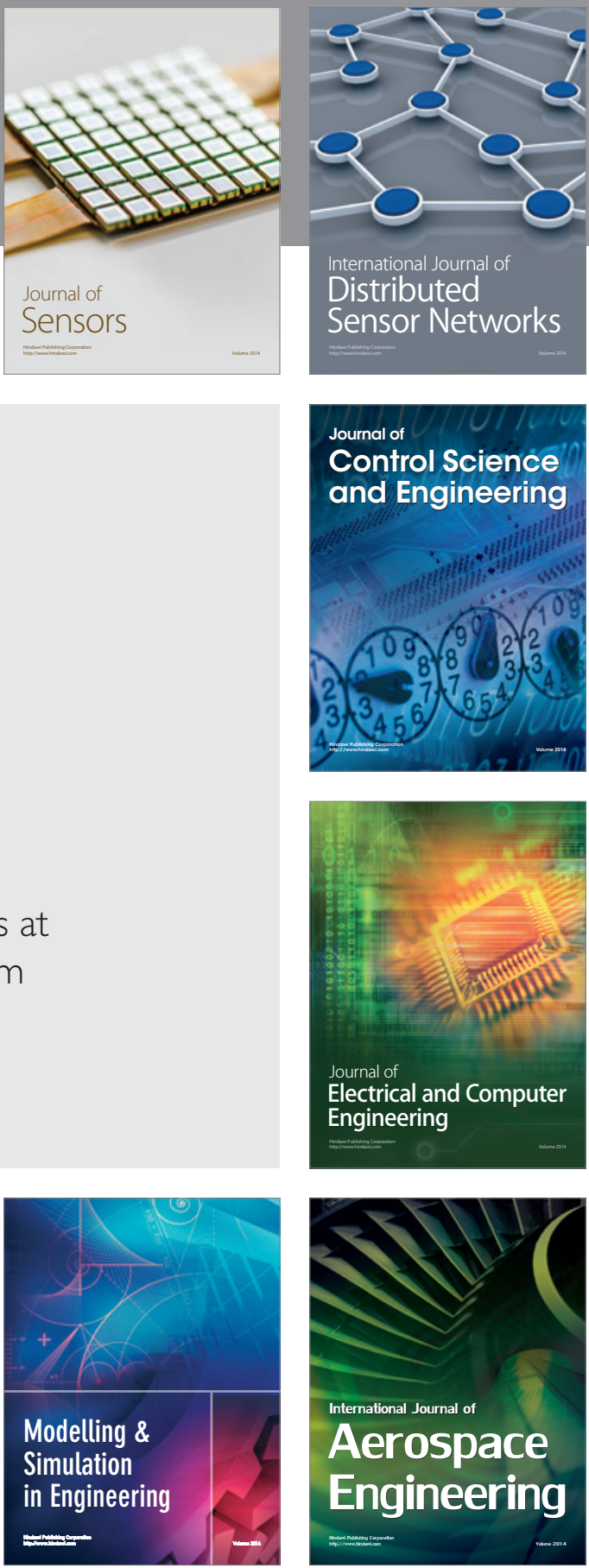

Journal of

Control Science

and Engineering
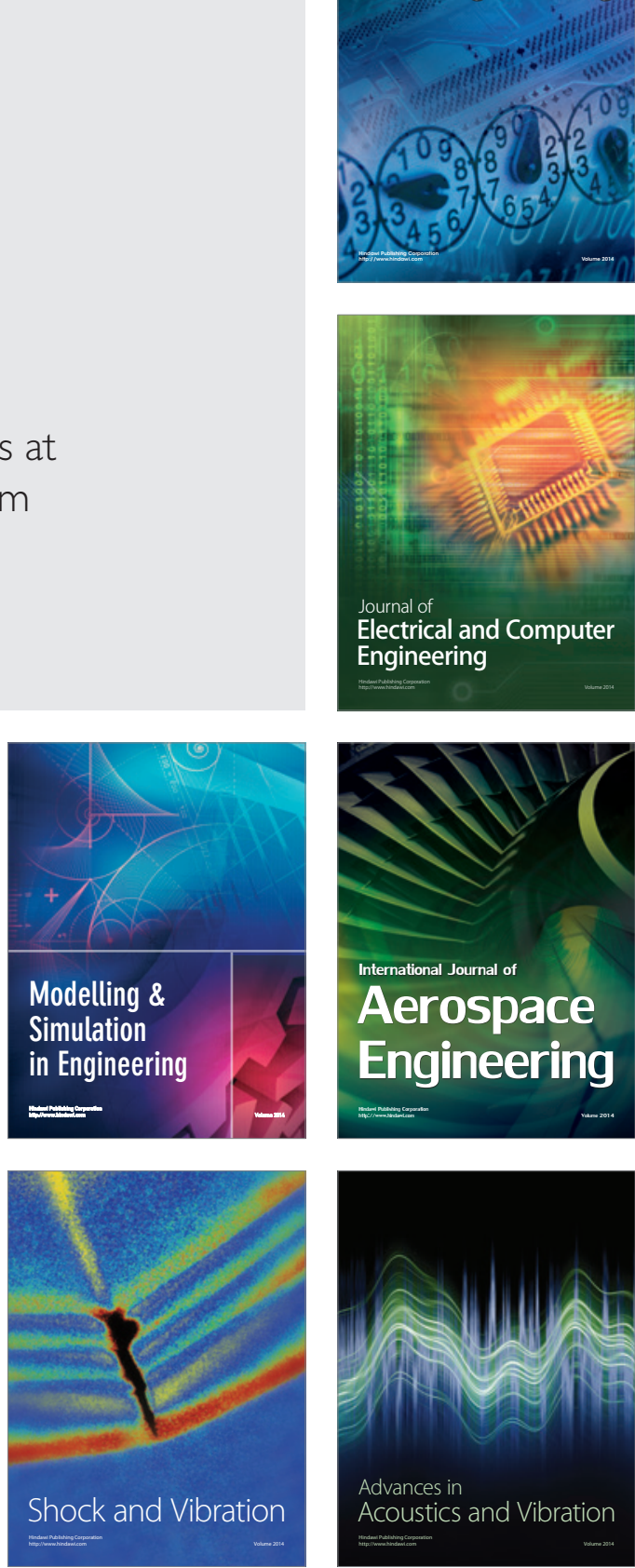